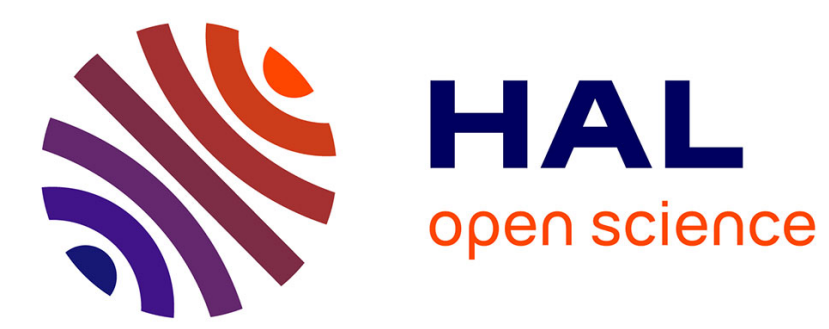

\title{
Le monde des morts
}

Claude Raynaud

\section{To cite this version:}

Claude Raynaud. Le monde des morts. Gallia - Archéologie de la France antique, 2006, Antiquité tardive, haut Moyen Âge et premiers temps chrétiens en Gaule méridionale (première partie) : réseau des cités, monde urbain et monde des morts, 63, pp.137-156. 10.3406/galia.2006.3293 . hal-01914302

\section{HAL Id: hal-01914302 https://hal.science/hal-01914302}

Submitted on 8 Jan 2020

HAL is a multi-disciplinary open access archive for the deposit and dissemination of scientific research documents, whether they are published or not. The documents may come from teaching and research institutions in France or abroad, or from public or private research centers.
L'archive ouverte pluridisciplinaire HAL, est destinée au dépôt et à la diffusion de documents scientifiques de niveau recherche, publiés ou non, émanant des établissements d'enseignement et de recherche français ou étrangers, des laboratoires publics ou privés.

\section{(이) $\$$}

Distributed under a Creative Commons Attribution - NonCommercial - NoDerivatives 44.0 


\title{
LE MONDE DES MORTS
}

\author{
Claude RAYNAUD
}

\begin{abstract}
Mots-clés. Nécropole, sépulture, réutilisation, mobilier funéraire, tombe rupestre, sarcophage, christianisation.
Résumé. Au cours des dernières décennies, les fouilles de nécropoles ont opéré un renouvellement radical de la documentation concernant les pratiques funéraires en Gaule méridionale, autorisant la révision d'anciens paradigmes et imposant des interprétations plus nuancées que par le passé. Typologie, orientation et chronologie des tombes, dépôt de mobilier, modalités et contexte des réutilisations, topographie des champs funéraires, permettent de reconstituer une évolution lente et sensiblement plus complexe qu'on ne l'a longtemps cru. Ce renouvellement de la problématique tient aussi aux premières avancées de l'anthropologie biologique. Des questions majeures comme celles de la christianisation et de l'impact des " invasions » ne peuvent plus désormais être abordées sur un mode binaire et imposent un déplacement et un élargissement de la perspective en envisageant plus globalement le changement culturel. Malgré de forts décalages dans le développement des recherches, qui laissent dans l'ombre de vastes secteurs géographiques, l'ensemble des données tisse une trame d'une grande densité, à l'image de la complexité des mutations opérées au cours de l'Antiquité finissante.
\end{abstract}

Key-words. Cemetery, burial, reuse, grave-goods, rupestral tomb, sarcophagus, christianisation.

Abstract. During these last decades, burial excavations radically renewed the documentation concerning funerary practices in southern Gaul, allowing the reading of former paradigms and imposing more subtle interpretations than before. Burial typology, orientation and chronology, deposits, modes and context of reuse, topography of funerary areas, make possible the reconstruction of a slow and sensibly more complex evolution than it was for a long time thought. This new problematic is also due to the first developments of biological anthropology. Leading questions such as Christianisation and impact of invasions cannot be tackled on a binary pattern and impose different orientations and a widened perspective, in considering cultural change as a all. Despite strong gap differences in the increase of research, which leave in the dark some large areas, a very dense framework is being set by evidence being collected in the image of the complex mutations happened through the end of Antiquity.

Translation: Isabelle FAUDUET

Schlagwörter. Nekropole, Bestattung, Wiederbelegung, Grabausstattung, Sarkophag, Christianisierung.

Zusammenfassung. Die in den letzten Jahrzehnten durchgeführten Ausgrabungen von Nekropolen haben eine radikale Erneuerung unserer Kenntnis der Bestattungssitten im südlichen Gallien zur Folge. Damit wird jetzt die Revision alter Paradigmen möglich und nuanciertere Interpretationen als in der Vergangenheit werden provoziert. Informationen zu Typologie, Ausrichtung und Chronologie der Gräber, Beigabenausstattung, Modalitäten und Kontext von Wiederbelegungen, Topographie der Gräberfelder lassen uns eine langsame Entwicklung nachzeichnen, die spürbar komplexer ist, als man bisher glauben wollte. Der Neuansatz der wissenschaftlichen Fragestellungen ist auch auf die ersten Fortschritte der biologischen Anthropologie zurückzuführen. Zentrale Fragen wie die nach der Christianisierung und nach den Auswirkungen der "Invasionen » können fortan nicht mehr nach einem simplen "Ja-Nein-Schema " erörtert werden. Gefordert ist vielmehr ein Perspektivenwechsel und eine Horizonterweiterung im Hinblick auf einen Kulturwandel im weitesten Sinne. Mit dem höchst unterschiedlichen Forschungsstand bleiben zwar weite geographische Räume derzeit noch im toten Winkel. Entsprechend der Komplexität der kulturellen Veränderungsprozesse, die gegen Ende der Antike am Werk waren, ergeben die vorliegenden Daten aber nun ein äußerst dichtes Gefüge.

Übersetzung: Stefan WIRTH

En l'an 469, Sidoine Apollinaire narre dans une lettre à son fils la fâcheuse aventure survenue alors qu'il quittait Lyon pour rejoindre Clermont. " Il s'en est fallu de peu que dans la journée d'hier (oh! douleur !) une main sacrilège n'ait profané le tombeau de mon grand-père, ton arrière- grand-père; mais dieu était présent pour empêcher qu'une pareille impiété fût consommée. Le champ où il repose, rempli depuis des années tant de cendres funéraires que de corps, ne recevait plus depuis longtemps de nouvelles fosses» (Sidoine Apollinaire, Lettres, III, 12). L'exorde de 
Sidoine brosse un tableau de la situation: la tombe de l'aïeul court grand risque dans le champ des morts qui est en voie d'abandon, en cette seconde moitié $\mathrm{du} \mathrm{V}^{\mathrm{e}} \mathrm{s}$., au point que les fossoyeurs semblent avoir oublié la disposition d'anciens tombeaux. Dans ces lignes se trouvent ramassées les questions que l'on se pose sur le monde des morts et, à travers ces morts, sur l'eschatologie des temps paléochrétiens.

Chrétien, le grand-père l'était déjà vers la fin du $\mathrm{IV}^{\mathrm{e}} \mathrm{s}$. et il était le premier de la famille, ainsi que nous l'apprend la lettre un peu plus loin. Chrétien certes, mais encore pétri de culture classique, le poète pense d'abord, avant la grande parousie, au repos du défunt dans cette terre où il a trouvé sa dernière demeure, dans ce champ hors des murs de la cité, à l'écart de toute église. Sidoine Apollinaire précise encore le tableau lorsqu'il nous apprend : « [...] me rendant d'aventure à la ville de Clermont, j'aperçus du sommet de la colline voisine ce crime contre les lois de l'État. Me précipitant au galop de mon cheval, tant sur les pentes raides que sur le plat, [...] j'arrêtai, avant même d'être arrivé, par les cris qui me précédaient, l'audacieux forfait. » Peut-on être plus net? Le champ est bien visible depuis la voie publique car nul édifice, nulle clôture n'arrête le regard, nulle rumeur citadine ne trouble la quiétude du champ des morts ${ }^{67}$. La question se pose donc: la tradition antique est-elle pleinement respectée ou se survit-elle seulement? Si les lois de l'État s'appliquent toujours à préserver le lieu du sépulcre, le geste des fossoyeurs montre aussi que rien, plus rien n'indique dans le champ l'emplacement de la sépulture. Moment d'abandon, mais très progressif et sans interdit : on revient donc occasionnellement inhumer dans le vieux champ des morts?

«[...] Rempli depuis des années tant de cendres funéraires que de corps [...]»: simple jeu réthorique sur la cendre des défunts, évocation plus concrète des reliefs de banquets funèbres, ou bien doit-on lire à la lettre la réalité d'un champ où, depuis le Haut-Empire, s'entassaient les défunts, d'abord incinérés, les suivants inhumés ? On verra comment l'archéologie nous renseigne sur cette question de la continuité des pratiques funéraires. Quant au tombeau lui-même, il devait être bien discret ou bien dégradé pour que les humbles fossoyeurs aient cru pouvoir disposer des lieux. Pourtant Sidoine connaît bien, lui, l'emplacement, et

67. Il est bien difficile de suivre l'éditeur des Lettres, A. Loyen, lorsqu'il propose de localiser la sépulture dans l'une des nécropoles attenantes à un lieu de culte de la périphérie lyonnaise, l'église Saint-Étienne ou celle des Macchabées, aucune n'étant mentionnée par Sidoine Apollinaire (p. 224, note 34). il n'hésite pas un instant à préserver le locus religiosus devenu invisible. Humilité ou précarité des signes extérieurs de la sépulture ? Là encore, l'archéologue doit s'interroger.

«Je confesse mon erreur: je ne pus remettre à plus tard le châtiment de mes prisonniers mais je flagellai ces brigands sur la sépulture même de notre aïeul, autant que l'exigeaient la piété des vivants et la sécurité des morts ». L'eschatologie antique s'affirme dans ce souci de préserver l'intégrité de la tombe: le double du défunt est bien là, dans la tombe où il poursuit une vie atténuée; dérangé, il deviendrait redoutable pour le repos des vivants. En ce $\mathrm{V}^{\mathrm{e}} \mathrm{s}$. domine une sensibilité traditionnelle; c'est seulement la génération suivante qui, très progressivement et avec de longues hésitations, recevra le message d'Augustin et placera son espérance en la cité de Dieu, où l'âme délivrée n'a que faire de la dépouille qui gît dans la fosse. Une mort païenne donc? Mais le mot a-t-il un sens avant que ne s'impose, à rebours, une mort chrétienne?

Pourtant, la suite ne laisse aucune ambiguité sur le contexte: l'évêque domine désormais la vie sociale et, sitôt après avoir donné cours à la colère, Sidoine lui adresse une missive, s'excusant de ce qu'il a empiété sur sa justice et soulignant habilement qu'il a agi ainsi afin «que l'évêque n'eût à sévir avec une rigueur incompatible avec sa dignité ». Ainsi l'évêque punit-il désormais, garant du droit dans la cité, quoiqu'en ces temps l'Église naissante n'exige ni n'interdit rien en ce qui regarde les pratiques funéraires. Hors de toute règle écrite, l'archéologie éclaire cependant l'attraction qu'exercent désormais sur les morts les édifices chrétiens : si la tombe de l'aïeul se trouve en un lieu désolé, c'est que désormais l'attention se porte sur les inhumations ad sanctos, au plus près de Dieu et de ses saints. Sidoine le sait bien, et s'il n'est pas encore évêque lors des faits, ne songe-t-il pas déjà à élire pour sa sépulture le circuitus de quelque église, en ville ? Dans les lignes qui suivent, s'affirme en effet l'abandon de la sépulture aux champs : « [...] afin de rendre impossibles pour l'avenir des accidents que ce précédent nous fait un devoir d'éviter, je demande que sur le champ [...] la masse de terre dispersée soit relevée en forme de tertre que recouvrira une plaque polie ». Le délaissement de l'ancien champ des défunts laisse craindre en effet de nouveaux accidents, que l'on tente de prévenir en réalisant une épitaphe. «De mon côté, j'ai laissé au vénérable Gaudentius le prix de la pierre et le coût du travail. Les vers qui suivent et que j'ai composés la nuit dernière sont loin, je crois, d'être brillants, car je ne manquais pas d'être préoccupé par le voyage. Si médiocres qu'ils soient, je te demande pourtant de les faire rapide- 
ment graver sur la plaque; mais veille à ce que le graveur ne fasse pas de faute dans le marbre; la faute qu'il pourrait commettre, soit à dessein soit par inattention, le lecteur jaloux me l'attribuerait plutôt qu'au lapicide. Si tu veilles, avec un pieux empressement, à l'exécution de ce que je t'ai demandé, je t'en aurai, quant à moi, autant de reconnaissance que si ce geste ne t'apportait pas à toi aussi un supplément d'honneur et de gloire [...]». Vieille habitude que cette célébration écrite, destinée autant à la mémoire du défunt qu'à la notoriété de ses descendants, qu'il importe de signifier au passant. Ainsi se perpétuent les pratiques de la piété ostensible qui depuis les siècles de la République entouraient la sépulture. Bien loin d'un discours officiel sur la sépulture, discours inexistant au demeurant, la missive de Sidoine nous renseigne sur une sensibilité et sur l'expression d'une piété familiale n'ayant que faire de la religion.

L'inscription de ces pratiques sociales dans l'espace funéraire, nous la retrouvons dans une autre missive de Sidoine lorsque, cette même année 469, il narre une amicale assemblée à l'occasion de la fête de saint Just, à Lyon (Lettres, $\mathrm{V}, 17)$. La scène se déroule près de la basilique dans laquelle vient de s'achever le service des vigiles. Sidoine et ses amis se rafraîchissent auprès du tombeau du consul Syagrius, dans un tout autre cadre que celui de la lettre précédente. Les amis devisent à l'ombre de la treille qui orne le tombeau, près de la maison du gardien : image de l'otium dans ce cadre bucolique, pourtant ancré dans la réalité de son temps, près du tombeau du saint. Entretenue, jalousement gardée, la tombe de Syagrius ne craint pas la profanation.

Ostentation, tradition, religion : subtilement, les lettres de Sidoine tissent le voile à travers lequel nous apparaît, partielle et troublée, une image de l'homme devant la mort, dans cette Antiquité finissante dont l'archéologie peut dévoiler certains traits. De la signalisation du tombeau à la topographie funéraire et à la topographie chrétienne, Sidoine Apollinaire trace ainsi un itinéraire, entre le poids du passé et les signes du renouvellement. Suivons-le, dans le domaine des realia qui seul nous est accessible mais qui, en bien des points, confirme et amplifie les propos du poète.

$\mathrm{Au}$ cours de cette enquête, prenons garde cependant de ne pas nous égarer dans l'insignifiance qui guette l'accumulation des données matérielles. Dans sa spontanéité, le récit de Sidoine insiste sur les questions qui faisaient agir et réagir $a u v^{e}$ s., sur ce qu'il était nécessaire de préserver : l'intégrité du sépulcre dans son environnement agreste. De l'architecture tombale, des dépôts funéraires, rien n'est dit : parce que cela importait peu, parce que ce n'était pas en jeu, parce que chacun faisait à son goût ? C'est bien difficile à dire, mais cela doit immuniser contre la tentation de trop attendre de la typologie des tombes, de l'organisation des dépôts ou de la parure du défunt, qui sont avant tout les outils de l'archéologue pour classer, dater et comparer, et dont on peine souvent à percevoir le sens. À rebours, ira-t-on jusqu'à dénier tout contenu à l'idée de "coutume funéraire » et à rejeter ces gestes, pieusement accomplis, dans l'insignifiance du folklore ? La difficulté est bien, dans ces temps où aucune règle n'était fixée par écrit, de déceler derrière ces gestes privés, une idée de la mort.

\section{HISTORIQUE DES RECHERCHES}

Dans le Midi comme ailleurs, l'archéologie funéraire fut longtemps cantonnée à la collecte du mobilier funéraire. Cette approche s'inscrivait dans une perspective diffusionniste qui dominait l'historiographie du XIX ${ }^{\mathrm{e}} \mathrm{s}$. et de la première moitié $\mathrm{du} \mathrm{XX}^{\mathrm{e}} \mathrm{s}$. : il importait avant tout de suivre l'installation des peuples germaniques. Significativement, le travail fondateur de Barrière-Flavy était une Étude sur les sépultures barbares, assortie d'un sous-titre tout aussi révélateur puisqu'il s'agissait de traiter de l'Industrie wisigothique (Barrière-Flavy, 1893). Et de fait, si l'on mentionne encore l'ouvrage de ce pionnier c'est essentiellement pour ses belles gravures présentant le mobilier funéraire. Des sépultures, il n'était guère question, quand les méthodes de fouille restaient fort expéditives et ne permettaient guère autre chose que de faire de belles trouvailles. On connaît les limites de cette archéologie illustrative qui, n'ayant d'autre objet que d'étayer par l'exemple les conclusions de l'histoire textuelle, reste démunie de toute approche critique de ses propres sources.

$\mathrm{Au}$ début du $\mathrm{XX}^{\mathrm{e}} \mathrm{s}$, , la discipline sort du cabinet des antiquaires pour entreprendre une classification des différents modes de sépulture par la méthode stratigraphique à partir de l'exemple, malheureusement sans lendemain, de la nécropole de Saint-Baudille-le-Vieux à Nîmes (Mazauric, 1915). Mais c'est seulement après la Seconde Guerre mondiale que se multiplient les recherches et qu'est entrepris un premier "essai de chronologie typologique » concernant Les Sépultures à inhumation du III ${ }^{e}$ au XIII ${ }^{e}$ siècle de notre ère dans la basse vallée du Rhône (Gagnière, 1965). Après les approximations des précédentes décennies, s'impose désormais une classification rigoureuse, au détriment toutefois de l'analyse sociologique et de l'anthropologie culturelle. En effet, les questions d'interprétation paraissent résolues avant d'être posées, ainsi que l'indique la récurrence, dans les études des décennies 1930 à 1960, de la notion de «sépulture 
barbare ». Pourquoi une sépulture est-elle «barbare »? Parce qu'elle est contemporaine ou postérieure aux grandes migrations, quoi que l'on puisse dire sur « les nombreux exemples de monnaies impériales romaines trouvées dans les tombeaux barbares » (id., ibid., p. 54). Dans cette étude qui fit longtemps référence, si le problème de la coutume est abordé, c'est seulement à propos du passage de l'incinération à l'inhumation, "peut-être sous l'influence des religions orientales, pour triompher plus tard avec le christianisme » (id., ibid., p. 55). Depuis le XIX ${ }^{\mathrm{e}}$ s. en effet, s'impose à l'archéologue une lecture étroitement religieuse des données funéraires qui ne sont guère analysées qu'à travers des réminiscences liturgiques.

L'élargissement de l'approche intervient seulement dans les années 1980. Après l'ample perspective ouverte par P. Ariès sur l'histoire de L'Homme devant la mort (1977), P.-A. Février revisite le thème de la mort à l'époque paléochrétienne et dans le haut Moyen Âge, dans un profond travail où il met en lumière la complexité des phénomènes (Février, 1977, 1978a, 1981, 1983b, 1984, 1987). S'attachant à distinguer ce qui, autour de la tombe, reste dans la pratique sociale et ce qui a trait au religieux et au symbolique, P.-A. Février invite à relativiser la portée des observations archéologiques, bien modestes en regard des actes symboliques et des gestes entourant le départ du mort, pratiques qui ont peu de chances de laisser quelque trace matérielle. Il insiste au contraire sur la nécessité de refonder la démarche en évitant de projeter les idées des liturgistes sur les observations archéologiques. L'une des principales leçons à retenir est celle de la continuité de certaines croyances antiques, tout autant que de pratiques sociales ayant peu à voir avec la religion et qui « témoignent d'une anthropologie commune aux païens comme aux chrétiens »(Février, 1987, p. 945).

Cette approche d'anthropologie culturelle se développe au cours des années 1980 et prend toute sa place dans une synthèse consacrée aux Sépulture et traditions funéraires $d u V^{e}$ au $X I I I^{e}$ s. apr. J.-C. dans les campagnes des Alpes françaises du Nord (Colardelle, 1983). Ainsi s'affirme une analyse affranchie de l'ancienne référence religieuse, les gestes funéraires étant lus désormais comme des pratiques sociales. De cette lecture émerge une perception du temps de la mort qui s'inscrit dans la continuité du temps de la vie, dans la perspective d'un enracinement de l'habitat qui, à la fin de l'Antiquité, se fixe durablement. Plus récemment encore, dans son approche de la topographie funéraire, l'archéologie éclaire un effet de cette sédentarisation accrue, de cette «territorialisation » de la sensibilité : comme la maison et moins éloignée qu'on ne l'a longtemps cru, la tombe appartient aux générations successives occupant la ferme, la villa ou le village. À l'image classique de vastes "nécropoles» rassemblant la population à la ronde, les travaux récents ajoutent la nuance, sensible, de petits groupes sépulcraux restés au plus près de l'habitat. La mort antique est privée, familiale et sociale. Elle le restera longtemps.

P.-A. Février invitait à développer l'étude des cimetières, non seulement pour nourrir l'approche anthropologique de la mort mais aussi pour pallier les incohérences de la documentation sur le peuplement (Février, 1978a, p. 235). C'est un chapitre sur lequel, en effet, beaucoup a été fait au cours des deux dernières décennies, tout au moins sur le terrain. Les fouilles de nécropoles sont désormais prati-quées en tendant vers l'exhaustivité, ou au moins vers la représentativité des données, en se préoccupant de développer une approche globale incluant la topographie et la hiérarchie de l'habitat (fig. 72). C'est dans ce cadre qu'ont été entreprises des recherches de longue haleine associant à l'étude des habitats la fouille des nécropoles, à Larina (Hières-surAmby) (Porte, Buchet, 1985; Porte 2001) et à Lunel-Viel (Raynaud, 1987). Plusieurs synthèses régio-nales ont été entreprises, d'abord en Viennoise (Colardelle, 1983), puis en Narbonnaise première (Raynaud dir., 1987; Manniez, 1999), tandis qu'en Provence plusieurs études renouvellaient la documentation avec la publication de la nécropole de Cadarache, près de la Durance (Pouyé et al., 1994), la fouille partielle de Ménerbes (Cartron et al., 1992, 1995) et plus récemment la série des fouilles pratiquées sur la ligne du TGV (Blaizot et al., 2001; TGV Méditerranée, 2002). À ces données rurales s'ajoutent les informations révélées par les fouilles urbaines, notamment à Vaison-la-Romaine (Carru dir., 1991), Arles (Heijmans, 2003), Digne-lesBains (Démians d'Archimbaud, Pelletier, 1995), Marseille (Moliner dir., 2003; Rothé, Tréziny dir., 2005, p. 566-572) ou encore Narbonne (Ginouvez et al., 1997; Ginouvez, 1999) ainsi que sur l'île de Maguelone à Villeneuve-lès-Maguelone (Garnotel, 2004). Dans la haute vallée du Rhône, les études ont porté sur les édifices paléochrétiens de Lyon (Reynaud, 1998) et de Grenoble (Colardelle, 1986), aussi bien qu'à la campagne, à la faveur des fouilles préventives, près de Genève (Privati, 1986) ou plus largement entre l'Ain et l'Isère (Faure-Boucharlat dir., 2001).

Dans le Sud-Ouest enfin, si les nécropoles de Toulouse (Wild, 1999) et de Saint-Bertrand-de-Comminges (Guyon, Paillet, 1996) ont fait l'objet de travaux récents, c'est essentiellement à la campagne que se sont développées les fouilles, de Venerque (Vidal, 1987) à Beaucaire (Larrieu 


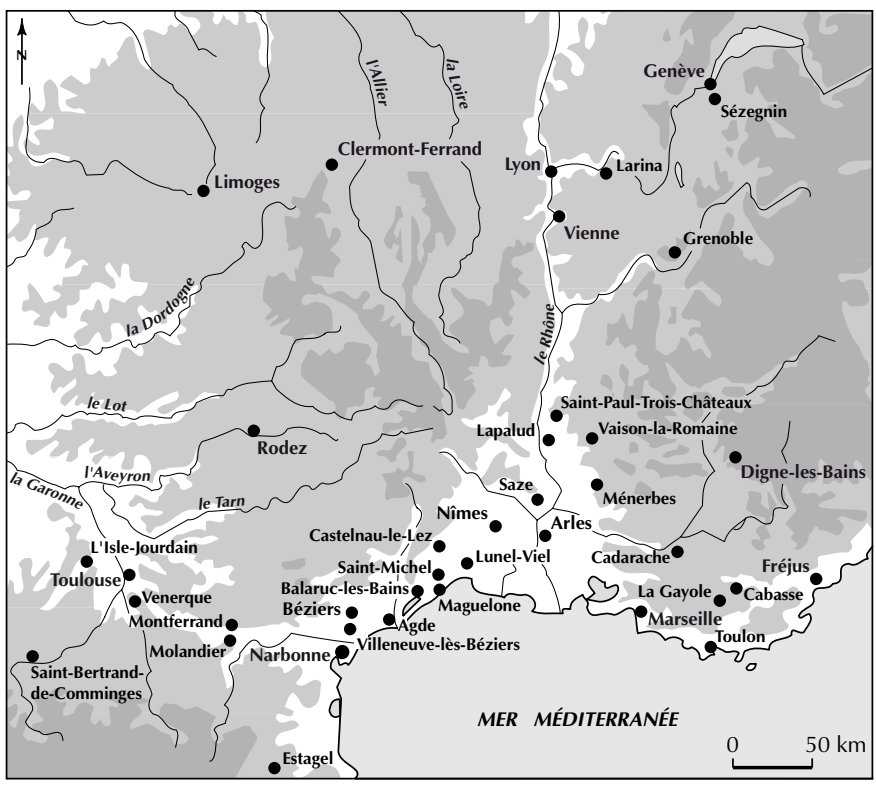

Fig. 72 - Localisation des principales nécropoles mentionnées, révélant un fort tropisme des recherches dans la vallée du Rhône, sur le littoral et dans le couloir Aude-Garonne. Le Massif central et les Alpes demeurent méconnus (DAO C. Raynaud, CNRS).

et al., 1985), et plus récemment à L'Isle-Jourdain (Bach, Boudartchouk, 1998), pour ne citer que les principales opérations. En Aquitaine enfin, si l'enrichissement des données paraît moins sensible, on dispose désormais d'un bilan succinct sur les nécropoles établies dans les ruines des villae (Balmelle, 2001).

Le déploiement de ces études archéologiques a suscité en premier lieu des études d'anthropologie biologique, qui ont nécessité un long travail de mise au point des méthodes et des objectifs, et qui restent encore trop limitées. Deux équipes animent ces recherches, l'une au sein du laboratoire d'anthropologie de Bordeaux, conduisant une projet collectif sur le Sud-Ouest (Crubézy dir., 1999) et des opérations préventives dans la vallée du Rhône (Blaizot et al., 2001), l'autre au Centre de recherches archéologiques de Sophia-Antipolis, à qui l'on doit les études sur la vallée du Rhône (Carru dir., 1991; Porte, Buchet, 1985). L'étude des nécropoles de Lunel-Viel vient toutefois nuancer les attentes fondées sur ces premières données paléodémographiques, la dégradation des os ne favorisant pas toujours le diagnostic biologique (Raynaud dir., à paraître a) .

Cet ensemble de travaux a sensiblement renouvelé la documentation, à commencer par la typologie et la datation des sépultures, complétées et affinées dans une synthèse concernant l'ensemble du Sud-Est (Colardelle et al., 1996).
Pour le reste, la documentation demeure éparse au gré des études monographiques, aucune synthèse n'ayant été entreprise jusqu'au présent dossier. De la topographie funéraire à la tombe, des coutumes funéraires aux rapports avec le monde des vivants, voyons point par point les avancées tout autant que les voies sans issue ou les pistes oubliées...

\section{NÉGROPOLE}

«Le champ où il repose [...]»; Sidoine Apollinaire nous rappelle opportunément que du temps de son grandpère, le champ des morts n'existait pas autrement que par les sépultures qu'il accueillait, et qu'il s'était développé hors de tout cadre formel : aucun mot ne s'attachait à le désigner. Quittant la route, notre cicerone gagne le lieu du sacrilège au galop de son cheval, sans avoir à franchir nulle clôture. C'est bien ainsi que nous apparaissent les champs funéraires lorsque l'on peut les fouiller amplement, à Sézegnin, à Lunel-Viel ou à Cadarache. L'affaire est entendue et il n'y a pas lieu de s'étendre : jusqu'au VI ${ }^{\mathrm{e}}$ ou VII ${ }^{\mathrm{e}}$ s., l'aire sépulcrale demeure dans la tradition antique, en plein champ, le long des voies d'accès, à l'écart de l'habitat.

Tradition n'est pas immobilisme, aussi ne doit-on pas s'étonner de l'extrême rareté, surtout à la campagne, des nécropoles à utilisation longue. On a envisagé une continuité à la nécropole de la Guérine à Cabasse, mais l'ensemble ne fut que partiellement fouillé, de sorte que l'on ne peut exclure une interruption après la période d'incinération, du I ${ }^{\mathrm{er}} \mathrm{s}$. au début du $\mathrm{III}^{\mathrm{e}} \mathrm{s}$., puis une reprise des inhumations à partir du $\mathrm{V}^{\mathrm{e}} \mathrm{s}$. (Bérard, 1980). Le cas est plus tranché dans la vallée du Rhône à Saze où, après les incinérations des $\mathrm{I}^{\mathrm{er}}-\mathrm{II}^{\mathrm{e}} \mathrm{s}$., les inhumations ne sont pratiquées qu'à partir du IV ${ }^{\mathrm{e}} \mathrm{s}$. (Gagnière, Granier, 1972). On pourrait multiplier les exemples de telles ruptures dont on doit se demander si elles expriment des mutations proprement funéraires, ou si elles sont au contraire le simple effet d'un autre processus, plus ample, touchant à l'ensemble du réseau de peuplement. Mais il est bien rare que l'on ait étudié simultanément nécropole et habitat, de sorte que cette vision d'ensemble fait généralement défaut. À LunelViel où le dossier est assez fourni, si le village n'a guère bougé on sait, en revanche, que la nécropole du Verdier n'était pas fréquentée avant les dernières décennies du $\mathrm{III}^{\mathrm{e}} \mathrm{s}$., après que la pratique de l'inhumation eût supplanté l'incinération. Pour sa part, la nécropole du Haut-Empire, dont témoignent six épitaphes découvertes anciennement, nous échappe encore malgré d'intenses recherches, ce qui ne permet pas de saisir la façon dont s'opéra le changement. 


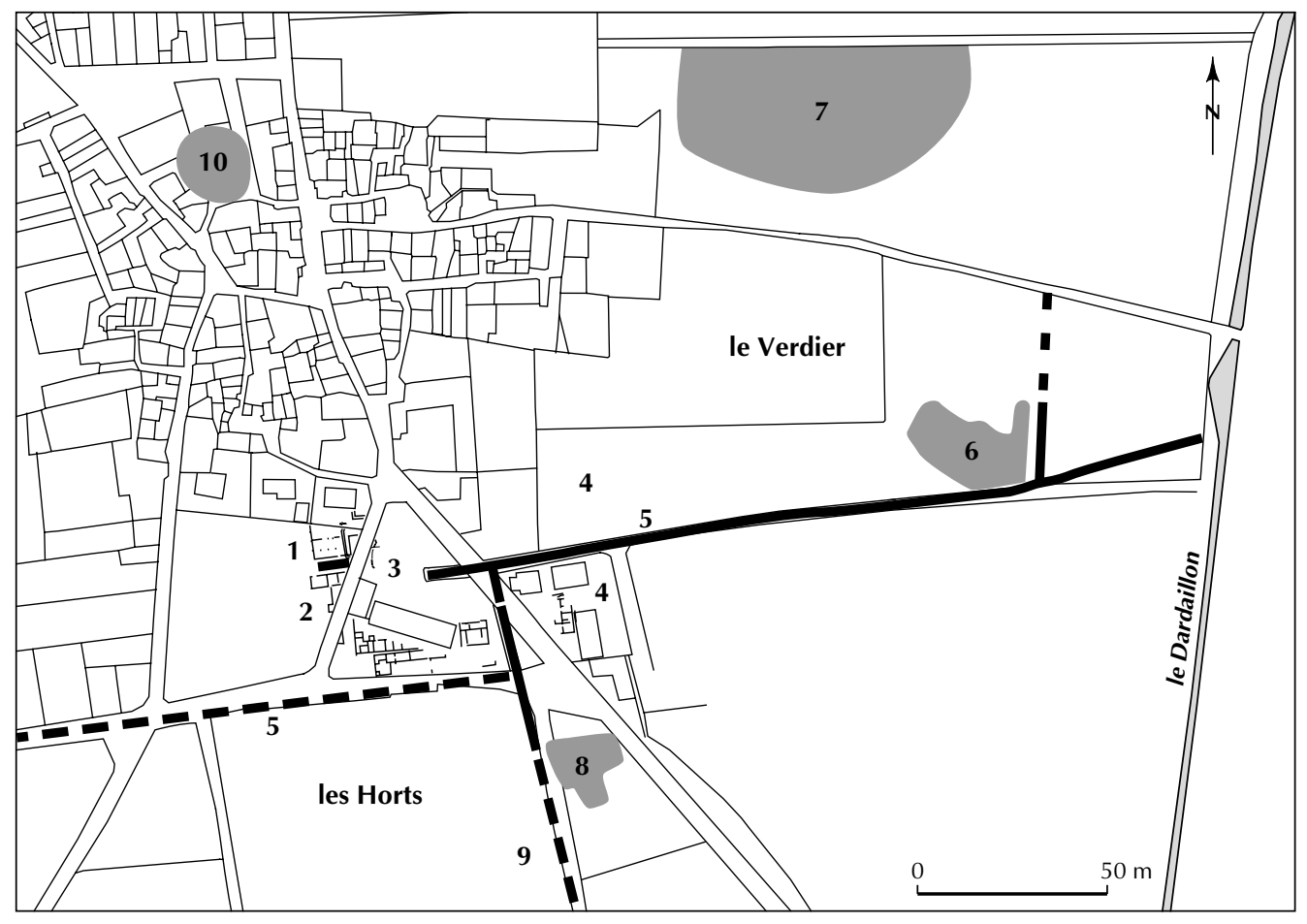

\begin{tabular}{|lll|}
\hline zone fouillée & zone restituée \\
\hline
\end{tabular}

Fig. 73 - Topographie funéraire d'une agglomération rurale. Les trois nécropoles de Lunel-Viel (Hérault) : 1, thermes de l'Ouest et palestre ; 2, quartier ouest de l'habitat; 3, quartier central gallo-romain ; 4, quartier sud; 5, chemin antique de la Carriérasse; 6, nécropole tardo-antique du Verdier; 7, habitat tardo-antique du Verdier; 8, nécropole wisigothique des Horts; 9, chemin antique des Horts; 10, habitat et nécropole de Saint-Vincent au haut Moyen Âge (DAO C. Raynaud, CNRS).

Par un ballottement dont l'archéologue doit s'accommoder, il faut donc aller à Saint-Paul-Trois-Châteaux pour observer le changement: à la nécropole du Valladas la mutation intervient lentement avec toute une série de formes intermédiaires. De premières inhumations concurrencent les incinérations dès le $\mathrm{I}^{\mathrm{er}} \mathrm{s}$., puis le phénomène s'amplifie aux II $^{\mathrm{e}}$ et $\mathrm{III}^{\mathrm{e}}$ s. (Bel, 2002). Malheureusement, à SaintPaul-Trois-Châteaux c'est la nécropole tardive qui n'est pas connue et la série reste incomplète une fois de plus !

Malgré tout, les exemples paraissent suffisamment concordants pour que l'on retienne l'idée d'une première vague d'abandon des nécropoles au cours du $\mathrm{III}^{\mathrm{e}}$ s., accompagnée (et/ou provoquée ?) par une évolution des pratiques funéraires. Une seconde "rupture », elle aussi vérifiée par de nombreux exemples, marque le $\mathrm{IV}^{\mathrm{e}} \mathrm{s}$. au cours duquel nombre de nécropoles se trouvent délaissées, sans que l'on doive conclure à l'abandon concomitant de l'habitat. À Lunel-Viel, où la documentation est la plus dense, vers le premier tiers du $\mathrm{VI}^{\mathrm{e}} \mathrm{s}$. la nécropole ne fait que se déplacer de quelques centaines de mètres et se dédouble même, entre le site des Horts et celui où - déjà ou plus tard ? - s'élève ou s'élèvera l'église Saint-Vincent (fig. 73). C'est un processus analogue que révèlent les fouilles de Larina à Hières-surAmby, avec l'abandon de la première nécropole, vers le début $\mathrm{du} \mathrm{VI}^{\mathrm{e}} \mathrm{s}$., au profit d'un nouveau champ funéraire autour de l'église, sur la colline du Mollard (Porte, 2001) (fig. 74). Comme pour la rupture du III $^{\mathrm{e}}$ s., les causes de ce déplacement, qu'aucune contrainte topographique n'imposait, sont à chercher du côté des mentalités et des schémas culturels, peut-être aussi du côté de la religion. Sans invoquer l'action de l'Église qui ne s'occupera que fort tard d'encadrer les morts (Treffort, 1996), on ne peut s'interdire d'envisager de nouvelles préoccupations à l'égard de la sépulture que l'on souhaite peut-être désormais retrancher de l'ancien cadre païen. Dans des campagnes, où la documentation ancienne laissait soupçonner l'éclosion de cimetières autour des églises rurales, le phénomène pouvait être envisagé dans la perspective d'une genèse 


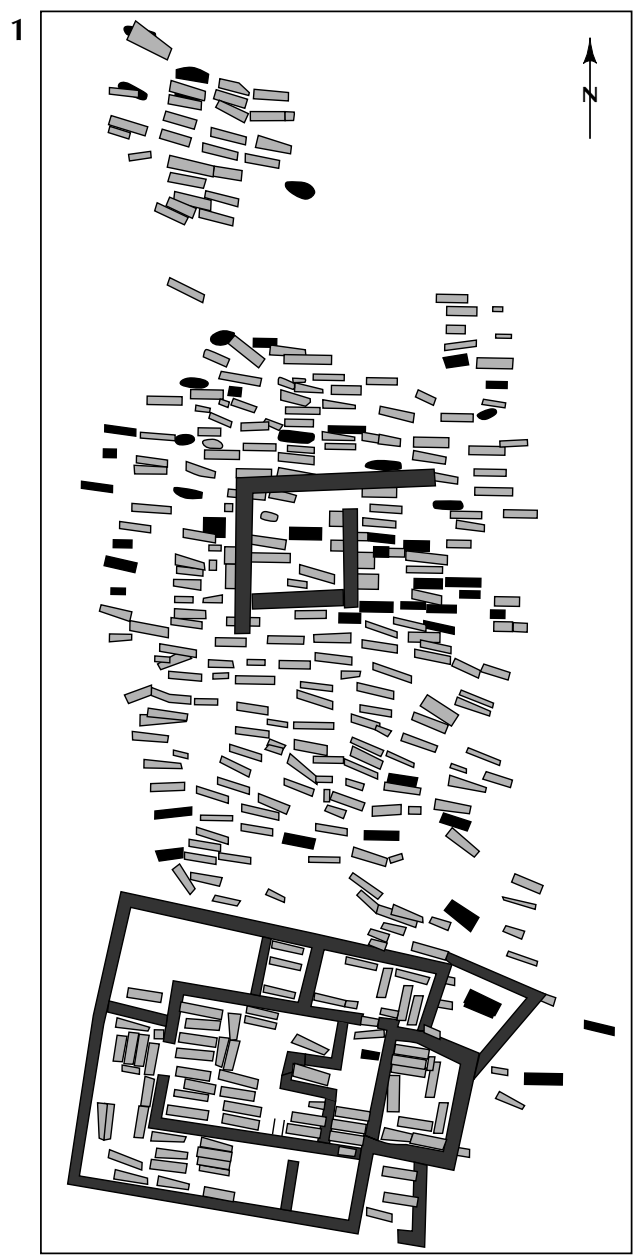

$\underline{0}$ $10 \mathrm{~m}$

$\square$ coffre en dalles $\square$ indéterminé tombe en fosse $\quad$ ZZA tombe sous tuiles $\because \because$ zone détruite

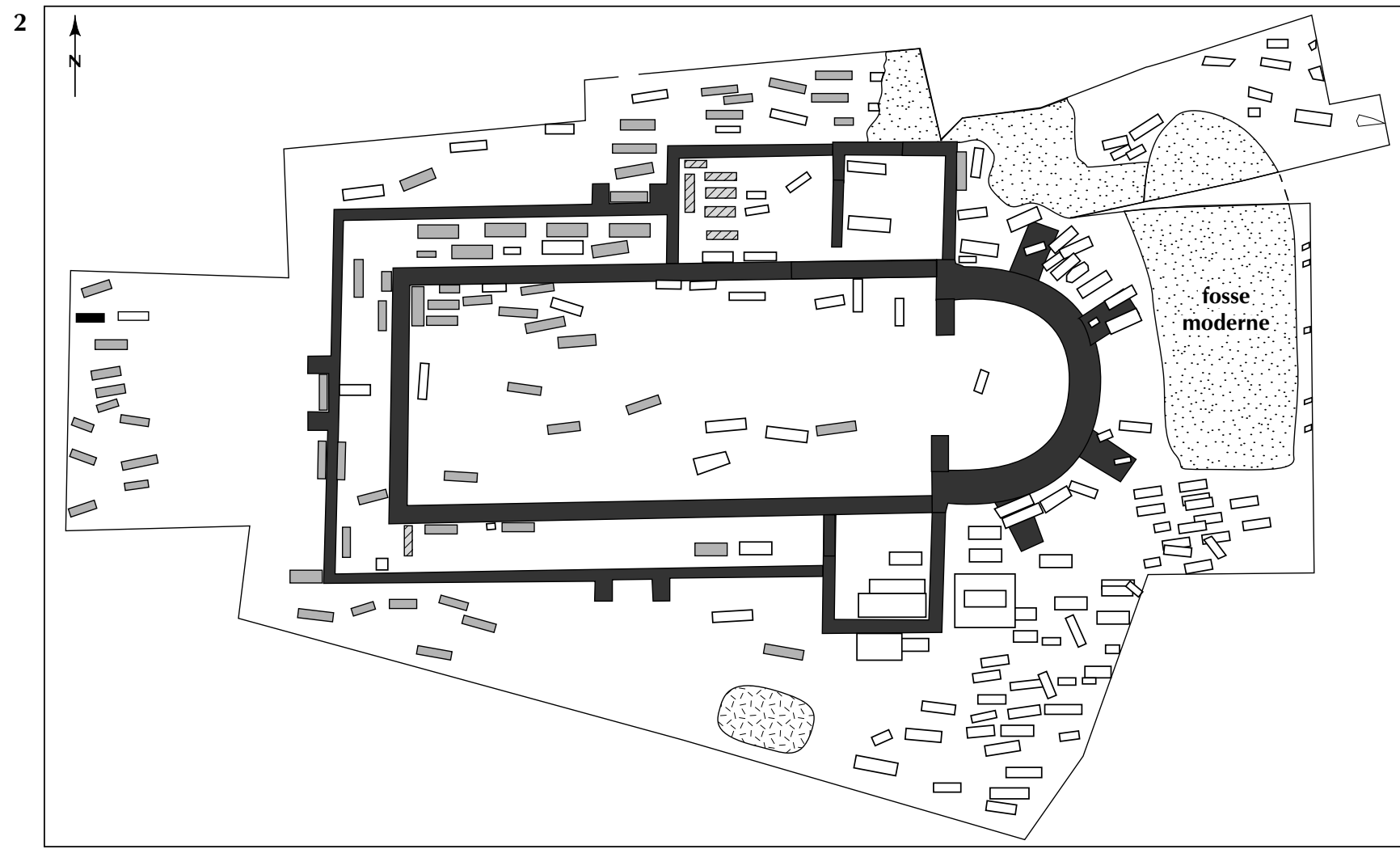

Fig. 74 - Plans comparés des nécropoles autour des édifices religieux des VI ${ }^{e}$-VII ${ }^{e}$ s. :

1, le Molard à Larina (Hières-sur-Amby, Isère) ; 2, Maguelone (Villeneuve-lès-Maguelone, Hérault) (DAO A. Garnotel, CNRS). 
du cimetière chrétien (Février, 1978b, p. 236-237). L'idée était nourrie par le constat d'un groupement de plus en plus sensible des tombes autour des églises, tant à la ville qu'à la campagne, à partir du $\mathrm{V}^{\mathrm{e}} \mathrm{s}$. L'ambitieuse entreprise conduite sur la topographie chrétienne de Lyon (Reynaud, 1998), tout autant que les fouilles d'édifices paléochrétiens (Duval dir., 1995) ne laissent aucun doute à cet égard : avec l'église, les morts font leur entrée en ville (Galinié, Zadora-Rio dir., 1996). Mais cette archéologie du cimetière chrétien reste insuffisante, le phénomène apparaissant de façon plus nuancée depuis que l'on mesure la quantité des sépultures, isolées ou groupées, qui échappaient encore à tout environnement religieux. C'est là une contribution majeure de l'archéologie préventive qui, en imposant de vastes décapages autour des habitats, a élargi la perception du phénomène. À Dassargues, près de Lunel-Viel, dès le $\mathrm{VI}^{\mathrm{e}} \mathrm{s}$. un groupe de sépultures s'établit à proximité de l'habitat, dans les champs au bord de chemins (Garnier et al., 1995). Un copieux dossier, d'intérêt majeur par la qualité de son élaboration, a pu être rassemblé sur d'autres fouilles réalisées dans la vallée du Rhône à l'occasion de la construction du TGV Méditerranée et de l'autoroute A46 (Blaizot et al., 2001; Maufras dir., 2006, p. 281-362), ainsi que dans la région lyonnaise (Faure-Boucharlat dir., 2001). Une vingtaine d'ensembles s'étalant $\mathrm{du} \mathrm{IV}^{\mathrm{e}} \mathrm{au} \mathrm{X} \mathrm{X}^{\mathrm{e}} \mathrm{s}$. ont été étudiés de manière exhaustive et sont précisément localisés dans leur environ-nement agraire. Rassemblant généralement de cinq à trente sépultures établies en quelques décennies seulement, ces groupes funéraires n'apparaissent plus désormais comme un fait marginal ou une survivance, mais bien au contraire comme un trait dominant de la topographie funéraire, jusqu'aux abords de l'an mil. C'est après seulement que l'on peut parler, en toute certitude, de l'affirmation du cimetière chrétien dans un contexte paroissial qui s'affirme alors comme un encadrement plus strict (Treffort, 1996).

Le bilan est plus décevant en ce qui concerne la question des rapports sociaux. Si au Haut-Empire l'éventail des richesses se reflétait, au moins en partie, dans les sépultures, de tels signes n'émergent guère dans les nécropoles tardives qui, avec leur mobilier stéréotypé, laissent " peu de place à l'identité sociale », particulièrement à la campagne (Feugère, 1993, p. 155). Même en ville l'éventail n'est guère marqué, à l'exemple des sarcophages non décorés de la nécropole du Jardin d'Hiver, à Arles, qui contenaient de modeste bijoux d'or et des poteries communes (Sintès dir., 1987, p. 121-123). À Marseille toutefois, des circonstances exceptionnelles permettent de corriger cette image terne. Dans la nécropole de Saint-Victor, le contraste frappe entre le beau sarcophage du sacrifice d'Isaac et la modestie du mobilier qu'il contenait, mais la conservation exceptionnelle du vêtement révèle une parure de tissus coûteux, marque de richesse qui aurait échappé à notre attention dans des conditions de conservation moins favorables et, hélas, plus courantes (Boyer, Fattori, 1976). Les tombes des élites, c'est une évidence, se trouvent ailleurs, par exemple $\mathrm{du} \mathrm{IV}^{\mathrm{e}} \mathrm{au} \mathrm{VI}^{\mathrm{e}} \mathrm{s}$. dans le monument funéraire de la Gayole à La Celle (Démians d'Archimbaud, 1971). Les chercherat-on en ville, autour de la cathédrale ? L'exemple du siège épiscopal de Maguelone apparaît peu convaincant, où les sépultures fouillées ces dernières années ne se distinguent guère de celles du village de Lunel-Viel (Garnotel, 2004). Tous ces éléments sont connus mais ils apparaissent épars, sans lien. Ce qui manque c'est de saisir dans une ville, dans un terroir ou dans une région, l'étagement précis des fortunes et des statuts, dans son expression funéraire tout autant que dans sa topographie. Cette topographie sociale de la mort nous échappe encore.

La topographie de la nécropole a longtemps retenu l'attention des chercheurs qui observaient une nette différence entre les nécropoles des $\mathrm{IV}^{\mathrm{e}}$ et $\mathrm{V}^{\mathrm{e}} \mathrm{s}$. où les tombes, orientées tantôt dans l'axe nord-sud, tantôt dans l'axe est-ouest, se répartissaient sans ordre et se chevauchaient fréquemment, et les aires plus tardives où les tombes, toutes disposées est-ouest, s'alignaient en rangées régulières (pl. I et II hors texte). Il était loisible de discerner une nette césure entre les premiers cas, interprétés comme des nécropoles gallo-romaines, et les seconds où l'on supposait une influence ou une présence barbare. En réalité, cette opposition reposait sur des observations partielles et elle s'est trouvée démentie par le développement de fouilles extensives, à Lunel-Viel, à Villeneuve-lès-Béziers ou ailleurs, l'étude de grands ensembles révélant l'apparition, bien plus précoce que prévue, de rangées de tombes dès le $\mathrm{V}^{\mathrm{e}} \mathrm{s}$., au sein même de nécropoles « gallo-romaines" (Manniez, 1999, p. 35-37). L’organisation par rangées apparaît désormais comme un processus lent et continu, qui émerge tard, en effet, dans les nécropoles tardo-romaines et domine dans les nécropoles postérieures, sans que l'on puisse pour autant conclure à un changement brutal. Changement culturel, ethnique ? Effet à retardement de l'installation des migrants, ou simple rationalisation de l'espace funéraire ? Reconnaissons que le choix de l'une ou l'autre hypothèse relève plus de l'intime conviction que de la stricte démonstration. 


\section{FUNÉRAILLES}

Sur le départ du mort, l'archéologie a peu à dire : toilette, lamentations, prières, gestes d'accompagnement et protection magique nous échappent. Si ce chapitre est évoqué ici c'est en réalité pour souligner une absence, un silence sur le seul aspect que l'archéologie peut aborder, celui du repas funèbre. Abondamment illustrée par la littérature, l'épigraphie et l'iconographie, la tradition antique du repas funèbre, renouvelé à l'occasion des fêtes anniversaires, se perpétue durant l'Antiquité tardive. En témoignent de nombreux vestiges de tables et de lits érigés autour de la tombe, les mensae découvertes en Italie, en Afrique et en Tarraconaise. Le mort participe à ces libations, ainsi que le montrent les conduits ménagés dans les dalles ou les mosaïques, ou dans les catacombes, ces coupes scellées sur la sépulture, et si Augustin fulmine contre de telles pratiques, c'est surtout pour en dénoncer les débordements somptuaires ou éthyliques (Février, 1977 et 1981).

Si je rappelle ces données c'est pour souligner à rebours leur surprenante discrétion en Gaule méridionale où il faut conduire une quête minutieuse pour recueillir de bien maigres observations. De mensae, les seuls témoins matériels sont ceux que l'on a mis au jour dans la nécropole de Pommerol, à Vaison-la-Romaine, et qui sont datés du $\mathrm{V}^{\mathrm{e}}$ ou du VI ${ }^{\mathrm{e}}$ s. (Carru dir., 1991, p. 50). À Lyon, les fouilles de Saint-Just ont livré pour leur part des dépotoirs interprétés comme reliefs de repas funèbres (Reynaud, 1998, p. 205). À Arles, où l'iconographie des sarcophages paléochrétiens offre pourtant une image de ces repas funèbres (Guyon, Heijmans dir., 2001, p. 58), les fouilles n'ont livré aucun vestige matériel, non plus qu'à Narbonne. C'est à Toulon que l'on a découvert, disposés au-dessus de sépultures à bâtière de tuiles, des cols d'amphores africaines sectionnés qui pouvaient former des conduits à libation, permettant au mort de partager le repas des vivants. Les fouilles étaient malheureusement limitées, dans une rue de la ville moderne, et n'offraient pas l'ampleur nécessaire pour comprendre la topographie des lieux, de sorte que l'on reste bien en peine de conclure à la réalité de ces repas (Brun, 1999, p. 825-827). Tous ces exemples concernent la ville, mais l'on a des témoignages comparables à la campagne, par exemple près de l'agglomération de Balaruc-les-Bains (Pellecuer, 1986b, p. 110-114). Dans ces gestes qui préludaient à la séparation des vivants et des morts, peut-être faut-il aussi ranger l'unique exemple, dans la nécropole du Verdier à Lunel-Viel, d'une coupe en céramique déposée au-dessus d'un cercueil du IV ${ }^{\mathrm{e}} \mathrm{s}$., qui témoigne peut-être de libations au cours des funérailles, alors que la fosse n'était pas encore comblée (Raynaud dir., à paraître a) .

Absentes ou effacées ailleurs, ces marques restent bien minces pour que l'on précise cet accompagnement du défunt dans sa dernière demeure. Ainsi les populations de Narbonnaise, comme leurs voisines de Viennoise, de Novempopulanie et d'Aquitaine, honoraient cette tradition méditerranéenne, tant en ville qu'à la campagne.

\section{PASSAGE}

Une fois manifestées toutes les marques de piété à l'égard du défunt, il incombe aux vivants d'assurer au mort un passage dans l'au-delà. À cette fin, l'ancienne pratique de l'incinération, qui dominait à la période républicaine et sous le Haut-Empire, se trouva progressivement abandonnée au cours du III $^{\mathrm{e}}$ s., mais on connaît encore, de façon très exceptionnelle, un cas d'incinération au $\mathrm{IV}^{\mathrm{e}} \mathrm{s}$. dans la nécropole du Cirque à Arles, et un autre trouvé anciennement à Nîmes. Cette évolution est très largement attestée dans le monde romain, mais la façon dont s'est opéré le changement, son rythme même, demeurent mal cernés dans le sud-est de la Gaule où les données restent peu nombreuses en ce qui concerne ces siècles de mutation. Une enquête a recensé dans ces régions seulement 143 tombes attribuables au III $^{\mathrm{e}}$ s., dont un bon tiers autour de la ville de Lyon, ce qui donne une idée de l'ampleur des lacunes et du déséquilibre documentaire. Cette enquête a montré que la pratique de l'inhumation, attestée dès le $\mathrm{I}^{\mathrm{er}} \mathrm{s}$., reste très minoritaire jusqu'au milieu du $\mathrm{II}^{\mathrm{e}} \mathrm{s}$., date à laquelle elle connaît un rapide accroissement, jusqu'à devenir prépondérante au $\mathrm{III}^{\mathrm{e}} \mathrm{s}$. (Bel, Manniez, 1996). Si l'incinération est encore ponctuellement attestée dans la seconde moitié du III ${ }^{\mathrm{e}}$ s., c'est de façon tout à fait résiduelle, par exemple à Ambrussum (Villetelle) ou à Narbonne (id., ibid., p. 94).

Les $\mathrm{II}^{\mathrm{e}}$ et $\mathrm{III}^{\mathrm{e}}$ s. sont marqués par de nombreux abandons d'établissements et par la restructuration des réseaux d'habitat rural, dont les pôles principaux, agglomérations ou villae, assurent une certaine continuité (Raynaud, 1996). Rien n'autorise à penser que cette transformation des structures du peuplement et de la production agricole a pu jouer un rôle dans l'évolution des pratiques funéraires, mais dans un tel contexte on ne peut s'étonner de la rareté, surtout à la campagne, des nécropoles à utilisation longue, seules à même d'éclairer le déroulement du processus. On envisage une telle continuité de façon exceptionnelle à la 
nécropole de la Guérine à Cabasse, mais l'ensemble n'est que partiellement fouillé et l'on ne peut exclure une interruption après la période d'incinération $d u \mathrm{I}^{\mathrm{er}} \mathrm{s}$. au début $\mathrm{du} \mathrm{III}^{\mathrm{e}} \mathrm{s}$., puis une reprise des inhumations à partir du $\mathrm{V}^{\mathrm{e}} \mathbf{s}$. (Bérard, 1980). La rupture est nette en revanche dans le cas de Lunel-Viel où la nécropole du Verdier, fréquentée à partir des dernières décennies du $\mathrm{III}^{\mathrm{e}}$ s., n'a pas livré une seule incinération sur plus de 360 sépultures, et c'est un cas général que l'on retrouve dans toutes les nécropoles fouillées largement, de Villeneuve-lès-Béziers à Cadarache, de Frontignan à Sézegnin.

La continuité était-elle plus marquée autour des villes? C'est ce que laisseraient entrevoir les nécropoles de Fréjus, Arles ou Lyon, où l'on observe le groupement d'inhumations tardives à proximité de monuments du Haut-Empire. Mais ce que l'on note dans ces cas, c'est l'absence ou la minceur des données concernant la seconde moitié du $\mathrm{II}^{\mathrm{e}} \mathrm{s}$. et le $\mathrm{III}^{\mathrm{e}} \mathrm{s}$., de sorte qu'il faut envisager une rupture ou seulement un déplacement entre les deux phases sépulcrales (Béraud et al., 1985; Tranoy, 1987; Sintès dir., 1990). Il faut bien avouer que, dans la plupart des cas, les surfaces fouillées demeurent - à l'échelle de la ville - insuffisamment représentatives. Saint-Paul-Trois-Châteaux et Marseille au quartier Sainte-Barbe, constituent les heureuses exceptions où l'étendue des fouilles permet d'observer le passage à l'incinération dans toutes ses nuances : la mutation s'opère lentement avec de premières inhumations qui concurrencent les incinérations dès le $\mathrm{I}^{\mathrm{er}} \mathrm{s}$, , puis une amplification de la pratique, avec toute une série de formes intermédiaires, intervient aux $\mathrm{II}^{\mathrm{e}}$ et $\mathrm{III}^{\mathrm{e}} \mathrm{s}$. (Bel, 2002; Moliner dir., 2003). Malheureusement, à Saint-Paul-Trois-Châteaux la nécropole de l'Antiquité tardive n'est pas connue et la série reste incomplète, ce qui ne permet pas de suivre le processus jusqu'à son terme. La situation n'est guère différente à Marseille où quelques sépultures tardives se trouvent encore dans la nécropole de Sainte-Barbe, sans que l'on puisse interpréter l'évolution : la nécropole n'estelle plus fréquentée qu'occasionnellement, à l'image de ce qu'évoquait Sidoine Apollinaire à Lyon, ou bien la zone d'inhumation s'est-elle seulement déplacée localement?

Malgré les lacunes maintes fois soulignées, les exemples paraissent suffisamment concordants pour que l'on retienne l'idée d'une mutation des pratiques funéraires au cours du $\mathrm{III}^{\mathrm{e}} \mathrm{s}$. Partout la mutation s'opère très progressivement durant près de deux siècles pendant lesquels inhumation et incinération coexistent en un même espace funéraire. Puis vient un temps de rupture lorsque l'on choisit, dans les dernières années du III $^{\mathrm{e}}$ s., un nouveau lieu de sépulture où va se développer la nécropole des $\mathrm{IV}^{\mathrm{e}}-\mathrm{V}^{\mathrm{e}} \mathrm{s}$. À Lunel-Viel par exemple, où l'ampleur des décapages opérés offre une large vision, on comprend bien que le changement de lieu n'était imposé par aucune contrainte topographique : le moment venu, le choix fut fait de rompre avec l'antique nécropole à incinération. Le processus se déroule avec un arrière-plan culturel dans lequel on a souvent cherché un indice de christianisation (Gagnière, 1965, p. 55). Si l'on doit, suivant la leçon de P.-A. Février, écarter une lecture trop liturgique des observations archéologiques, on ne peut pour autant évacuer ce contexte religieux qui joua un rôle sur l'évolution des mentalités. Cela étant, les données archéologiques ne permettent de trancher d'aucune façon: si l'iconographie des sarcophages délivre parfois un message religieux, il faut bien dire que jusqu'au VI ${ }^{\mathrm{e}} \mathrm{s}$, , les signes matériels chrétiens demeurent absents dans la tombe, en ville comme à la campagne. Le problème s'avère d'autant plus complexe que, à côté de la mutation opérée, s'affirment maints signes de continuité des pratiques gallo-romaines.

\section{TOMBE}

Au premier rang de ces continuités se range la construction de la tombe qui, dans sa technique comme dans ses matériaux, se conforme aux types anciens. Le développement des fouilles a sensiblement corrigé la chronologie et complété la typologie des tombes au cours des dernières années, de sorte que l'on perçoit mieux désormais la permanence des techniques, tant vers l'amont, dans le Haut-Empire où plongent les racines, que vers l'aval où s'affirme le legs tardo-antique. Sans entrer dans le détail de ces questions qui ont fait l'objet d'une récente mise au point collective (Colardelle et al., 1996), on doit noter à l'égard de la chronologie un recours plus fréquent aux datations par radiocarbone, au cours des dernières années. Longtemps impraticable en raison de son coût et de son imprécision, cette technique a sensiblement précisé ses résultats et supplée de plus en plus souvent à un mobilier funéraire qui demeure peu explicite ou souvent absent. À l'égard des méthodes traditionnelles de datation, par le mobilier et/ou la typologie, on ne saurait trop insister sur la plus grande vigilance requise à l'égard de la durée d'utilisation ou de réutilisation de certaines pièces de mobilier, parure comme céramique. À Lunel-Viel par exemple, on peut citer la présence d'un vase en sigillée de la Graufesenque dans une sépulture qui ne peut être datée antérieurement au $\mathrm{IV}^{\mathrm{e}} \mathrm{s}$., ou encore une boucle de ceinture du IV $\mathrm{I}^{\mathrm{e}} \mathrm{s}$. dans une sépulture datée par le ${ }^{14} \mathrm{C}$ de la fin $d u \mathrm{~V}^{\mathrm{e}} \mathrm{s}$. ou du début du 
$\mathrm{VI}^{\mathrm{e}}$ s. (Raynaud dir., à paraître a). Quant à la stratigraphie, même dans les nécropoles où la densité des inhumations a entraîné des superpositions et des recoupements, cette méthode ne livre pas autre chose qu'une chronologie relative, aux intervalles fort lâches.

Coffres et bâtières de tuiles, coffrages de maçonnerie et cercueils en bois, bien attestés dans les sépultures à incinération depuis le $\mathrm{I}^{\mathrm{er}} \mathrm{s}$., dominent encore au $\mathrm{IV}^{\mathrm{e}} \mathrm{s}$. dans la plupart des nécropoles. La seule évolution notable tient à la forme de la tombe, allongée pour répondre à sa nouvelle fonction: contenir le corps inhumé (fig. 75). De même l'amphore, longtemps utilisée comme urne cinéraire, conserve-t-elle son usage funéraire. Les amphores africaines cylindriques convenaient particulièrement à la sépulture de nouveau-nés et de jeunes enfants, mais on connaît aussi quelques exemples d'adultes inhumés de la sorte, notamment à Marseille ou à Toulon, la tombe nécessitant alors deux amphores (Brun, 1999, p. 136-137; Rothé, Tréziny dir., 2005, p. 566-572). Le plomb aussi, déjà utilisé pour les coffres des incinérations, reste en usage pour les cercueils, quoique de façon limitée, probablement en raison de son coût: un seul exemplaire est attesté parmi les 360 sépultures du Verdier à Lunel-Viel. Cette nécropole, fouillée in extenso, donne une idée de la répartition des types du $\mathrm{IV}^{\mathrm{e}}$ au VI ${ }^{\mathrm{e}} \mathrm{s}$. Avec près de $40 \%$ le cercueil représente le type dominant, principalement dans les premières décennies de la fréquentation où il doit représenter plus de $70 \%$, tandis qu'il devient résiduel dès le $\mathrm{V}^{\mathrm{e}} \mathrm{s}$. Le coffre en tuiles vient au second rang sur l'ensemble de la fouille avec $13 \%$, mais là encore il faut pondérer en notant que ce type est surtout fréquent dans la seconde moitié du IV ${ }^{\mathrm{e}} \mathrm{s}$. et au début du $\mathrm{V}^{\mathrm{e}} \mathrm{s}$. Avec un chiffre voisin (12\%), la bâtière de tuiles appelle le même commentaire avec peut-être une plus forte représentation au $\mathrm{V}^{\mathrm{e}} \mathrm{s}$. Viennent ensuite les types minoritaires : amphores $(7,5 \%)$, tombes rupestres anthropomorphes $(5 \%)$, tombes rupestres simples $(4,6 \%)$, coffres mixtes de tuiles et de bois (3\%), cuves maçonnées $(2,2 \%)$, coffres de bois $(1,3 \%)$ et pleine terre avec un cas unique.

Cette litanie pourrait s'appliquer à bon nombre d'autres exemples, moyennant quelques modifications parmi les fréquences. Ce qu'il importe en effet de noter, c'est la forte homogénéité des architectures tombales à l'échelle d'une aire géographique, des Alpes aux Pyrénées, de la mer Méditerranée au Massif central.

À côté des types devenus classiques, on a pu identifier une forme longtemps méconnue, la sépulture rupestre en roche meuble (Raynaud, 1987, p. 126; Colardelle et al., 1996, p. 291-293). Les sépultures se présentent dans des

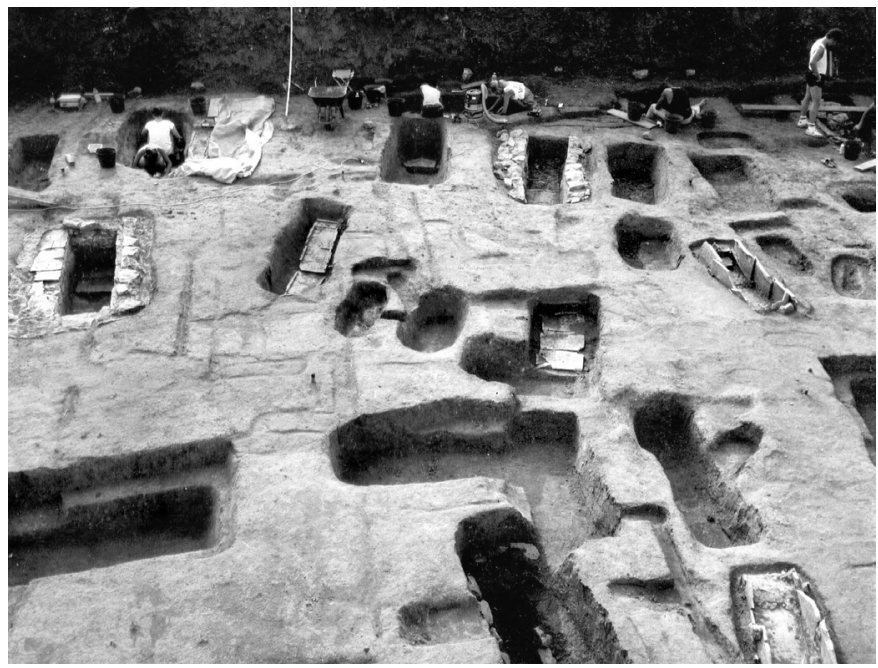

Fig. 75 - Le Verdier à Lunel-Viel (Hérault). Vue partielle de la nécropole avec des tombes en cuve maçonnée et en coffres de tuiles (cliché C. Raynaud, CNRS).

fosses étroites, parfois anthropomorphes, restées en l'état de creusement sans apport d'aucun matériau exogène : ni pierre de calage, ni clou, ni tuile. Elles étaient couvertes par un rang de tegulae ou de dalles horizontales reposant sur une feuillure ménagée dans les parois de la fosse à mi-profondeur. La taphonomie des sujets inhumés s'avère déterminante pour identifier cette pratique, en attestant d'une décomposition du corps en espace vide: maintien de tous les os dans l'espace initial du corps et déplacement des connexions labiles. Longtemps attribué exclusivement à la période romane dans le sud-est de la Gaule, le type rupestre est désormais attesté dès l'Antiquité tardive (Colardelle et al., 1996, p. 292-293). À Lunel-Viel, une série notable de sépultures a été attribuée aux $\mathrm{V}^{\mathrm{e}}-\mathrm{VI}^{\mathrm{e}} \mathrm{s}$. dans la nécropole du Verdier où l'une d'entre elles est datée au ${ }^{14} \mathrm{C}$ entre les années 540 et 665 , avec des pics de probabilité décroissante en 626, 624, 639, 605 et 585 (Ly-12052) (Raynaud dir., à paraître a). Le type est déjà attesté $\mathrm{au} \mathrm{IV}{ }^{\mathrm{e}} \mathrm{s}$. dans les nécropoles du Trillet, près de Lyon (Blaizot et al., 2001, p. 292-294), de Saint-André-de-Codols près de Nîmes (Pomarèdes dir., 1994, p. 158 et fig. 86), ainsi qu'au $\mathrm{V}^{\mathrm{e}}$ ou $\mathrm{VI}^{\mathrm{e}}$ s.; à Montboucher-sur-Jabron (Blaizot, 2002, p. 749), ou au Colombier à Vaison-la-Romaine (Carru dir., 1991). Loin d'apparaître comme un hapax sans postérité, les sépultures rupestres sont encore attestées dans la nécropole gardoise d'Aubais, avec une série de datations ${ }^{14} \mathrm{C}$ échelonnées entre la fin $d u V^{2}{ }^{e}$ s. et le $\mathrm{X}^{\mathrm{e}} \mathrm{s}$. (fouille M. Ott, inédit). La continuité du type rupestre-anthropomorphe est donc solidement établie depuis l'Antiquité tardive. Paradoxalement, ce 
sont désormais les sépultures attribuées à la période romane qui posent problème, dans la mesure où elles n'ont jamais fait l'objet de datations ${ }^{14} \mathrm{C}$ : il semble logique d'envisager à brève échéance une remise à jour des datations que l'on ne peut plus fonder sur la seule typologie.

Si j'ai insisté sur l'exemple des tombes rupestres, c'est à la fois pour souligner l'ampleur des mises à jour qui restent à opérer au sein de la documentation, tout autant que l'identification de nouveaux éléments d'une continuité culturelle entre basse Antiquité et haut Moyen Âge. Dans cette révision de la typologie, il convient de s'arrêter sur la sépulture en pleine terre. Ce fut longtemps un type dominant dans les nécropoles des $\mathrm{IV}^{\mathrm{e}}-\mathrm{V}^{\mathrm{e}} \mathrm{s}$., mais cette pratique s'avère en réalité peu fréquente depuis que les méthodes de fouille fine permettent de distinguer par la position des os et en particulier par l'observation des connexions labiles, les véritables dépôts en terre où le sédiment a immédiatement enrobé le cadavre, interdisant tout déplacement ultérieur, et les sépultures sous protection périssable, dans lesquelles les déplacements osseux trahissent une décomposition en espace vide, avant la désagrégation des pièces de bois. Ce type apparaît donc fréquemment surévalué, ainsi dans la nécropole genevoise de Sézegnin où une partie de ces tombes correspond à des coffrages de bois dont subsistaient seulement les pierres de calage (Privati, 1986, p. 23). La même confusion se pose dans la nécropole de Cadarache où la qualification de "pleine terre », qui concerne plus de $30 \%$ des tombes fouillées, recouvre en réalité, comme le notent les auteurs, des tombes rupestres à couverture de lauzes ou ayant perdu leur couverture (Pouyé et al., 1994, p. 74). La typochronologie des sépultures du sud-est de la Gaule signale l'existence de ce type du $\mathrm{III}^{\mathrm{e}}$ au V $\mathrm{e}$ s. dans la plupart des nécropoles de cette période, mais à l'image des tombes du Verdier, alors citées en référence, une large part de ces identifications n'apparaît plus recevable après les récentes mises à jour (Colardelle et al., 1996, p. 291).

L'exemple unique de Lunel-Viel invite même à s'interroger sur la portée de ce mode singulier: dans ce cas en effet, le défunt fut inhumé face contre terre, les mains liées dans le dos, posture tout à fait exceptionnelle et fortement connotée par l'idée de punition. À cet égard, il n'est pas inutile d'observer qu'au sein des tombes les plus finement analysées ces dernières années, les seuls dépôts en pleine terre identifiés dans la moyenne vallée du Rhône étaient réservés à deux chiens, dans la nécropole des Girardes, à Lapalud (Blaizot et al., 2001, p. 294-296). Est-ce assez souligner le caractère singulier de la tombe « en pleine terre ", que l'on ne peut plus identifier de façon anodine?
Cette typologie, bien établie dès les dernières décennies du $\mathrm{III}^{\mathrm{e}}$ s., demeure dominante jusqu'au milieu du V $\mathrm{V}^{\mathrm{e}}$ s. À cette date semble s'opérer une mutation progressive, d'abord marquée par des variations de détail: initialement très soignées, les sépultures sous tuiles - coffres ou bâtières - se font progressivement plus sommaires : au $\mathrm{V}^{\mathrm{e}} \mathrm{s}$., les tegulae tapissant le fond de la fosse disparaissent, généralement remplacées par des planches. Le bois est en effet de plus en plus présent, des planches remplaçant tout ou partie d'une paroi, d'un couvercle de coffre. Cette évolution aboutit à la diffusion du coffre en bois lorsque les tuiles disparaissent complètement. L'évolution s'observe partout, dès le $\mathrm{IV}^{\mathrm{e}} \mathrm{s}$. dans les régions alpines et la moyenne vallée du Rhône, seulement au $\mathrm{V}^{\mathrm{e}}$ et surtout au $\mathrm{VI}^{\mathrm{e}} \mathrm{s}$. dans les régions méditerranéennes. C'est là encore un processus lent qui s'opère sans bouleversement, contrairement à l'idée ancienne d'une introduction liée aux peuples barbares. Ce que l'on note à propos du bois, on peut l'étendre à l'égard des dalles ou des lauzes qui remplacent progressivement les tuiles au cours du $\mathrm{V}^{\mathrm{e}} \mathrm{s}$., avant d'aboutir à la construction de coffres complets, très répandus à partir $\mathrm{du} \mathrm{VI}^{\mathrm{e}} \mathrm{s}$. Le coffre en dalles est donc un type tardif à Lunel-Viel où il apparaît, comme le coffre en bois, au terme d'une lente transformation. On ne peut cependant généraliser cette observation au plan régional, dans la mesure où l'on connaît des exemples précoces dès le $\mathrm{IV}^{\mathrm{e}} \mathrm{s}$. en Vaunage (Gard), à quelques kilomètres de LunelViel (Parodi et al., 1987, p. 53) et surtout à Montpellier où la nécropole Saint-Michel en comptait onze exemplaires (Majurel et al., 1973). Révélant l'ampleur des décalages ou des contrastes parfois à l'échelle locale, ces exemples mettent en garde contre une confiance excessive dans les datations typologiques qui ont trop longtemps été privilégiées, au détriment des études de mobilier, de la stratigraphie et bien sûr du ${ }^{14}$ C. En ce sens, l'un des apports majeurs des travaux récents est d'affaiblir la démarche typochronologique en allongeant sensiblement les durées d'attestation des types, qui désormais doivent être croisés avec d'autres arguments pour fixer la datation des contextes funéraires.

Ce qui vient d'être évoqué à propos des coffres vaut aussi pour les bâtières dans lesquelles les lauzes ou le bois remplacent progressivement les tegulae, jusqu'à l'apparition de bâtières de lauzes, surtout fréquentes dans la basse vallée du Rhône à partir du VI ${ }^{\mathrm{e}} \mathrm{s}$. (Manniez, 1994), et de bâtières en bois, une variante plus rare dont on connaît seulement quelques exemplaires aux $\mathrm{VI}^{\mathrm{e}}-\mathrm{VII}^{\mathrm{e}} \mathrm{s}$., à Vienne et à LunelViel (Colardelle et al., 1996, p. 286). Quant à la datation, il en va de même que pour les coffres : pour ne donner qu'un exemple, emblématique, citons le cas des tombes 
sous bâtière de tegulae de la cathédrale de Digne, datées par ${ }^{14} \mathrm{C}$ du XI ${ }^{\mathrm{e}}$ s. alors que toutes les typologies s'accordaient jusqu'alors à faire de ce type un marqueur chronologique de la période paléochrétienne (Démians d'Archimbaud, Pelletier, 1995) ! Pour autant, la typologie des sépultures demeure un élément indispensable pour caractériser les faciès chronoculturels dans les nécropoles, et elle conserve une certaine valeur chronologique dans une majorité de cas, mais une majorité seulement: de nombreuses exceptions échappent aux pratiques dominantes qui ne furent jamais des règles absolues.

Dans cette typologie, on doit noter l'absence à ce jour de tombes à formae, constructions maçonnées à cuves juxtaposées. Les exemples catalans et italiens ne manquent pourtant pas, mais on ne connaît rien de tel en Narbonnaise, ni en ville ni à la campagne.

Tuiles, dalles, lauzes, bois, fosses rupestres : la typologie souligne la modicité des matériaux mis en ouvre dans la construction des tombes, tout autant que la simplicité des aménagements. Rurales ou urbaines, les nécropoles explorées sont dominées par les sépultures du commun, au sein desquelles il est bien difficile de saisir le reflet d'une hiérarchie sociale. Le sarcophage, qui conserve jusqu'au $\mathrm{VI}^{\mathrm{e}} \mathrm{s}$. son caractère ostentatoire avec son riche décor mythologique ou chrétien, reste réservé à la mince frange des classes dominantes. On le trouve seulement dans les domaines de l'aristocratie où les sépultures de la familia se rassemblent dans un monument, plus tard devenu chapelle funéraire : c'est l'exemple fameux de la Gayole à La Celle, autour du sarcophage d'Innodius Felix (Démians d'Archimbaud, 1971). Mais c'est essentiellement en ville que s'accusent les contrastes, comme l'illustre le cas d'Arles avec les sarcophages des nécropoles de Trinquetaille ou du Cirque, venus grossir ces dernières années la collection de la cité rhodanienne. Support paradoxal d'une ostentation que l'on cache dans la fosse, le sarcophage figuré perpétue jusqu'au $\mathrm{V}^{\mathrm{e}} \mathrm{s}$. une tradition classique réinvestie par les figurations chrétiennes (Février, 1979). Parallèlement, au $\mathrm{V}^{\mathrm{e}} \mathrm{s}$. la fameuse production d'Aquitaine introduit dans le décor une veine régionale dominée par le décor végétal et géométrique, traité avec un art consommé du semiméplat (Duval dir., 1993). Chrisme, canthare, colombe, paon, vigne : la symbolique chrétienne l'emporte désormais sur le legs classique dont la réminiscence se cantonne au décor architectural, pilastres et arceaux rythment encore le décor. Depuis l'épicentre de Saint-Béat, cette production a connu jusqu'au $\mathrm{VI}^{\mathrm{e}} \mathrm{s}$. une ample diffusion en ville comme à la campagne, de la Gironde à la Camargue, du piémont pyrénéen jusqu'à la vallée du Rhône, soulignant s'il en était besoin l'intégration des modèles culturels en Gaule méridionale, jusque dans le décor funéraire.

Par ces tombes de prestige, la familia honorait ses morts tout en affirmant son mérite et sa richesse, tandis que se pressaient autour les tombes anonymes. Cette mode aristocratique se diffusa cependant vers le bas lorsqu'au $\mathrm{V}^{\mathrm{e}} \mathrm{s}$., et plus encore $\mathrm{au} \mathrm{VI}^{\mathrm{e}} \mathrm{s}$., se développa une production de cuves anépigraphes et non décorées, attestées d'abord en ville, à Arles dans les enclos funéraires des Alyscamps (Heijmans, 1999, p. 165-166), à Narbonne (Ginouvez et al., 1997; Ginouvez, 1999) ou à Lyon (Reynaud, 1998, p. 214), pour ne citer que les exemples les mieux connus. Sur ces cuves parallélépipédiques, souvent munies d'un ressaut interne à l'emplacement de la tête, l'ornement se limitait au couvercle en bâtière dont les angles et l'axe médian portaient des ébauches d'acrotères, lointain souvenir du décor des $\mathrm{IV}^{\mathrm{e}}$ et $\mathrm{V}^{\mathrm{e}} \mathrm{s}$. L'étude des nécropoles de Lunel-Viel permet de suivre l'évolution en milieu rural : absents dans la nécropole $\mathrm{du}$ Verdier aux IV ${ }^{\mathrm{e}}$ et $\mathrm{V}^{\mathrm{e}} \mathrm{s}$., ces sarcophages - en tous points analogues à ceux que l'on observe en ville - apparaissent peu dans la première moitié $\mathrm{du} \mathrm{VI}^{\mathrm{e}} \mathrm{s}$. aux Horts, mais c'est surtout l'effet de pillages ( 5 cas pour 63 tombes identifiées) et ils sont en revanche bien représentés dans la seconde moitié de ce siècle dans la nécropole Saint-Vincent, où ils accueillent le tiers des sépultures. Dans un contexte encore mal cerné - domaine ou agglomération rurale ? la nécropole de Saint-Estève à Ménerbes a livré une série exceptionnelle par la diversité de son décor autant que par la prégnance de la symbolique chrétienne (Cartron et al., 1992). L'aire funéraire se singularise autant par sa localisation dans un bâtiment encadré d'une galerie - mausolée ou basilique ? - que par la densité inédite des sarcophages qui accueillaient 28 des 39 sépultures identifiées. Tout en suivant le modèle en bâtière à "pseudo-acrotères » précédemment décrit, les couvercles de ces tombes, sur lesquels se concentre l'essentiel du décor, se distinguent par la récurrence des signes chrétiens, laissés en réserve ou sommairement gravés : croix pattée, croix de Saint-André, tau (fig. 76). Dans un cas seulement, c'est la cuve qui porte, sur la paroi externe de son petit côté, une croix inscrite dans un cercle, en léger relief.

Ainsi se pérennisèrent jusqu'au $\mathrm{VI}^{\mathrm{e}} \mathrm{s}$., et parfois même au-delà, des gestes et des installations funéraires dont j'ai souligné l'ancienneté tout autant que les progressives mutations. Ayant peu à voir avec l'eschatologie et se tenant plutôt dans le champ social, ces pratiques se transmettront jusqu'au haut Moyen Âge. Voyons maintenant dans la tombe comment évolue le traitement des défunts. 


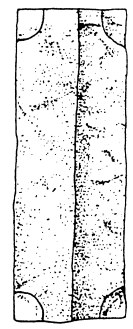

13

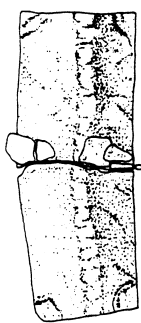

96

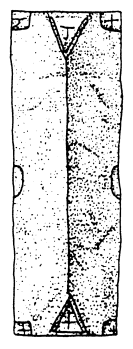

14

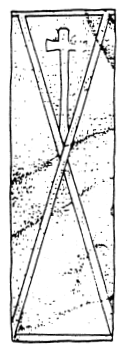

97

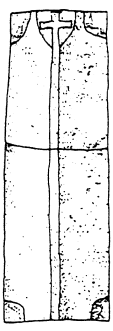

20

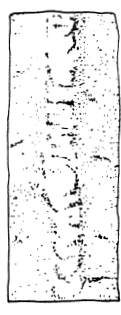

145

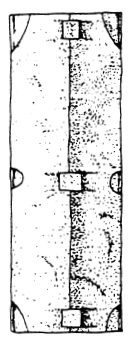

42

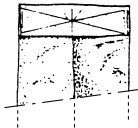

230
Fig. 76 - Ménerbes (Vaucluse). Couvercles de sarcophages paléochrétiens (d'après Cartron et al., 1992).

\section{SÉPULTURE}

Dans la tombe, le défunt était en règle générale allongé sur le dos, avec dans le détail quelques variantes sur la signification desquelles on s'interroge. La position des jambes, allongées, les pieds serrés ou légèrement écartés, et des bras, allongés de même, ou bien repliés sur le pubis ou sur l'abdomen, laissent envisager de multiples formes d'ensevelissement que seule une fouille attentive permet de distinguer : corps « libre » ou emmailloté dans un linceul, mains jointes ou écartées. À quelles croyances ou à quelles attitudes renvoient ces différentes pratiques? Cela reste difficile à cerner, d'autant que bien souvent la disposition varie sensiblement dans des sépultures voisines et contemporaines.

Plus rarement, le mort était déposé sur une litière que l'on peut identifier grâce au relevé rigoureux des clous lorsqu'ils se concentrent sous les ossements, grâce aussi à l'observation de certains déplacements d'ossements (Blaizot, 2002, p. 748-749). C'est le cas dans la nécropole du Verdier où l'on recense un seul cas, mais cette pratique demeure sous-estimée du fait de la difficulté d'identification, notamment dans les cas où la litière était assemblée à l'aide de chevilles.

Autre élément de caractérisation, l'orientation connaît elle aussi des variations dont il n'est pas aisé de saisir la signification. À Sézegnin comme à Lunel-Viel, où les fouilles extensives permettent d'étudier le développement topographique, la stratigraphie et la datation des sépultures révèlent un groupe de sépultures anciennes orientées nord-sud et fréquemment recoupées par des sépultures plus récentes, orientées quant à elles dans le sens est-ouest. Voilà une configuration banale, largement attestée dans l'ensemble des Gaules et au-delà. Les données chronologiques montrent que l'orientation nord-sud, prépondérante à la fin $d u \mathrm{III}^{\mathrm{e}} \mathrm{s}$. et au début $\mathrm{du} \mathrm{IV}^{\mathrm{e}} \mathrm{s}$., fut ensuite progressivement abandonnée au profit de l'orientation estouest qui devint exclusive probablement au deuxième quart $\mathrm{du} \mathrm{IV}^{\mathrm{e}} \mathrm{s}$. Dans un premier temps, la nouvelle pratique resta tâtonnante, comme on peut l'observer à Lunel-Viel : non seulement l'orientation de la fosse n'est pas rigoureuse - elle ne le sera jamais - mais encore la position du défunt varie, la tête tournée tantôt à l'ouest, tantôt à l'est, cette dernière position l'emportant seulement au $\mathrm{V}^{\mathrm{e}} \mathrm{s}$. Cette orientation, qui est restée celle de la sépulture pendant tout le Moyen Âge, fut un temps interprétée comme un indice de christianisation des pratiques funéraires, jusqu'à ce que l'on observe que cette mutation n'avait jamais été imposée ni même revendiquée par l'Église, et que de surcroît elle s'étendait au-delà du monde chrétien (Ariès, 1983, p. 14). Aucune interprétation convaincante n'apparaît donc en ce qui concerne ce choix dont le basculement est cependant trop systématique pour revêtir un caractère aléatoire.

J'a déjà évoqué, à propos de la tombe en pleine terre, le caractère exceptionnel de l'inhumation face contre terre, à Lunel-Viel dans la nécropole du Verdier. De rares exemples sont connus par ailleurs, par exemple à Castelnau-le-Lez, près de Montpellier (Vial, 2004, p. 152). S'agit-il là de la survivance d'une pratique fréquente dans les inhumations du Haut-Empire, à l'instar de la nécropole du Valladas, à Saint-Paul-Trois-Châteaux, où les corps étaient majoritairement déposés en procubitus, aux $\mathrm{I}^{\mathrm{er}}$ et $\mathrm{II}^{\mathrm{e}}$ s. (Bel, 2002, p. 103) ? Dans ce contexte préchrétien, une telle disposition pouvait témoigner d'une défiance à l'égard du double du mort dont il convenait d'empêcher le retour parmi les vivants. Le contexte était bien différent lorsqu'au $\mathrm{IV}^{\mathrm{e}} \mathrm{s}$. le défunt de la tombe 166 de Lunel-Viel fut inhumé en procubitus, mains derrière le dos, dans une fosse nord-sud et sans aucun mobilier. Cet ensemble d'indices, notamment le caractère exceptionnel de cette position ainsi que l'absence de mobilier dans la tombe, alors que les sépultures voisines en contenaient, incite à envisager une forme de punition pour un défunt dont on avait des raisons de se défier. 


\section{CULTURE, ETHNIE, CROYANCE ? LE MOBILIER FUNÉRAIRE}

Dans ce domaine encore s'impose la continuité avec le Haut-Empire. Le passage à l'inhumation n'a rien modifié dans l'habitude d'offrir ou de laisser au mort offrandes et objets personnels. Les faits sont connus dans leurs grandes lignes : jusqu'au $\mathrm{V}^{\mathrm{e}}$ s., le mort est accompagné par le mobilier du dernier repas, autant par égard pour le défunt que pour tranquilliser les vivants qui craignent toujours les revenants. À Lunel-Viel où la fouille du Verdier livre des chiffres significatifs, vaisselle, aliments et objets vestimentaires ou de parure composent l'essentiel de ces dépôts, souvent accompagnés d'une lampe. La quantité de ces dépôts varie d'une tombe à l'autre, mais des tendances s'opposent : tandis que $65 \%$ des tombes nord-sud, les plus anciennes, comportent du mobilier, la proportion descend à $30 \%$ dans les tombes est-ouest. De même les effectifs varient sensiblement entre le début du IV ${ }^{\mathrm{e}} \mathrm{s}$., où l'on peut trouver jusqu'à quatre à cinq poteries dans certaines tombes, et le début $d u \mathrm{~V}^{\mathrm{e}} \mathrm{s}$. où l'on ne compte plus qu'un vase, très occasionnellement, avant que les dépôts ne s'effacent complètement après le milieu $\mathrm{du} \mathrm{V}^{\mathrm{e}} \mathrm{s}$. Au sein d'un répertoire varié de vaisselle, les études relèvent habituellement la récurrence de l'association d'un vase à boisson, cruche, pichet, bol ou gobelet, et d'un vase à aliments, coupe, plat ou assiette, auxquels s'ajoutent accessoirement d'autres pièces, parfois redondantes : paires de bols ou de cruches, ou d'assiettes. À cette vaisselle s'ajoutent parfois les reliefs du dernier repas, déposés dans ou à côté des poteries. La nécropole des Clapiès, à Villeneuve-lès-Béziers, offre un large éventail puisque ces reliefs sont présents dans 53 sépultures sur un total de 74 tombes fouillées, ce qui représente une proportion bien supérieure à la plupart des nécropoles contemporaines (seulement 13 cas sur 360 à Lunel-Viel). Dans cet échantillon, les volatiles dominent de manière écrasante, suivis du mouton et du porc, du bœuf et du lapin, et enfin du poisson (Manniez, 1999, p. 162). À travers cet inventaire à la Prévert, représentatif d'un cas singulier parmi de nombreuses variantes, peut-être doit-on retenir la nette prépondérance des volatiles : cette domination relève-t-elle d'un choix, de la symbolique de l'oiseau ?

Les mêmes questions se posent à l'égard de ce que l'on nomme «l'inhumation habillée », pratique traditionnellement considérée comme un critère d'identification des nécropoles «barbares ». C'est un fait que les objets de parure se trouvent en proportion et en situation bien différentes dans les sépultures tardo-romaines où ils restent peu fréquents et généralement déposés avec les offrandes, tandis que dans les sépultures mérovingiennes ils apparaissent plus nombreux et sont portés par le défunt. Mais là encore la constitution de séries d'observations, en révélant la présence de mobilier d'habillement porté par le défunt dès le $\mathrm{V}^{\mathrm{e}} \mathrm{s}$., invite à nuancer l'opposition jusqu'alors trop franche et à envisager une progressive assimilation de la nouvelle «mode» (Feugère, 1993; Stutz, 1996). À Lunel-Viel par exemple, deux sépultures du Verdier offrent un contraste éclairant: tandis que dans la première, une plaque-boucle articulée se trouve déposée avec les poteries près des jambes du défunt, dans la seconde, une plaque-boucle analogue repose près de l'os iliaque, en position d'usage sur un vêtement. L'association avec des verreries situe ces tombes $\mathrm{au} \mathrm{V}^{\mathrm{e}} \mathrm{s}$. Mais la situation apparaît plus complexe car on ne peut exclure que la faible quantité des objets d'habillement, aux $\mathrm{IV}^{\mathrm{e}}$ et $\mathrm{V}^{\mathrm{e}} \mathrm{s}$., ne soit à mettre au compte d'un vêtement plus léger dépourvu d'éléments métalliques, les seuls que l'archéologue peut espérer retrouver. De la sorte, minoritaires $\mathrm{au} \mathrm{V}^{\mathrm{e}} \mathrm{s}$., les indices d'une inhumation habillée ne deviendraient nombreux aux $\mathrm{VI}^{\mathrm{e}}$ et VII ${ }^{\mathrm{e}}$ s., qu'au prix d'une distorsion.

L'étude des objets portés par les défunts débouche sur la question des populations barbares, apport exogène dont on peine à discerner l'ampleur. Rappelons d'abord que si ce mobilier provient essentiellement des sépultures, il ne s'agit en aucun cas d'une exclusive, ce que montre le développement des fouilles d'habitat où les découvertes ne manquent pas (par exemple Garnier et al., 1995, fig. 14; Mauné, Feugère, 1999, fig. 7). Seulement, il y a dans la tombe une notion d'ensemble clos et une mise en scène qui tendent à octroyer aux objets une valeur de signes forts. L'interprétation n'est pas toujours aisée, par exemple pour une plaque-boucle à décor excisé $d u \mathrm{~V}^{\mathrm{e}} \mathrm{s}$. découverte anciennement près de Montpellier, que l'on voudrait attribuer «à un soldat décédé sur place », un garde germain de l'usurpateur Constantin III replié sur Arles en 407 (Feugère, 1993, p. 146). Outre le fait que l'objet a pu être découvert dans un tout autre contexte que sépulcral, ce placage d'un indice archéologique sur un événement politique demeure fragile : rien n'interdit d'envisager une telle interprétation mais on n'en fera jamais la démonstration...

Dans le Midi gaulois, les recherches sur le mobilier d'habillement et de parure sortent d'une longue léthargie qui a succédé à la synthèse pionnière de James, en 1977. Des enquêtes rigoureuses se développent seulement depuis ces dernières années, invitant à reconsidérer certaines idées reçues, à commencer par la faible quantité du mobilier qui aurait rendu vain tout essai de synthèse (Feugère, 1993). 
En réalité, cette idée se trouve contredite par l'inventaire, pour la seule Septimanie, de près de 700 objets de parure (Hernandez, 2001). Une autre cause du blocage résidait dans la contradiction entre, d'une part, les influences diverses dont témoignent la technologie, la typologie et le décor de ces parures, et, d'autre part, la quête d'un faciès clairement défini. Cet obstacle s'estompe depuis que les études délaissent l'idée de dessiner des aires culturelles et ethniques aux contours étanches, pour aborder désormais le problème en termes de diffusion et de métissage. Un autre écueil encore résidait dans le concept de «costume national» adopté par les peuples barbares (Kazanski, 1992, p. 98). Si adéquat dans les régions d'Europe orientale d'où proviennent ces populations, ce concept s'est trouvé vidé de son sens au cours de la progression vers l'ouest, qui durant plus d'un siècle n'a pas manqué d'entraîner emprunts, échanges et influences. Au point d'arrivée de la longue migration, tous ces paramètres rendent vaine la quête de critères homogènes. On sait aujourd'hui qu'il n'existe aucun «faciès » wisigothique dans la culture matérielle, ni en Toulousain ni en Septimanie ni en Provence. Mais il apparaît tout autant que la diffusion des parures répond à des courants que l'on suit de mieux en mieux, du Toulousain marqué par des arrivages du domaine franc, à la Septimanie où les objets d'influence ou d'origine hispanique sont concurrencés par des apports du nord-est, tout autant que par un courant méditerranéen et byzantin (Feugère, 1988; Stutz, 1996; Hernandez, 2001). En Septimanie précisément, la chronologie de ces influences s'affine à mesure que s'étoffe la documentation. Un premier groupe de plaques-boucles à décor cloisonné manifeste peut-être l'éclosion, à la fin du $\mathrm{V}^{\mathrm{e}} \mathrm{s}$., d'un éphémère atelier régional (Kazanski, 1992, p. 94). Puis, au VI ${ }^{\mathrm{e}}$ s., les productions septentrionales semblent prendre le dessus, avec une fibule de Gondorf découverte à Maguelone, une autre de Thuringe à Lunel-Viel. L'imprégnation demeure toutefois relative et éphémère, avec le retour au VII ${ }^{\mathrm{e}} \mathrm{s}$. d'une "ambiance " plus méditerranéenne dont les parallèles se trouvent en Italie byzantine (Hernandez, 2001, p. 794; Hernandez, Raynaud, 2005).

Dans le Sud-Ouest, des fibules ansées de type germanique sont imitées à la fin $d u \mathrm{~V}^{\mathrm{e}} \mathrm{s}$. ou au début $\mathrm{du} \mathrm{VI}^{\mathrm{e}} \mathrm{s}$. (Feugère, 1988), puis un peu plus tard des bijoux à décor cloisonné (Stutz, 1996, p. 169). La situation est plus confuse en Provence où la faiblesse des découvertes - reflet d'une réalité ou manque de travaux? - obscurcit la perception des courants, tandis qu'au nord l'ancienne Viennoise est censée traduire un courant burgonde, dont la définition demeure incertaine (Colardelle, 1983, p. 373-380; Stutz, 1996, p. 169). Quant à la portée des tendances régionales, elle reste bien difficile à cerner : les cartes de répartition lacunaires traduisent-elles une présence réelle de migrants ou la simple diffusion des produits de quincaillerie et d'orfèvrerie ? Il est bien difficile d'y répondre tant que l'on raisonne sur des objets isolés ou sur des séries restreintes, les liens de cause à effet n'étant pas toujours aussi limpides qu'on le souhaiterait. Ainsi a-t-on remarqué que les objets de style wisigothique se diffusent en Toulousain majoritairement $\mathrm{au} \mathrm{VI}^{\mathrm{e}} \mathrm{s}$., alors que la région est « devenue » franque (Kazanski, 1992, p. 94)!

Si l'écheveau des influences et la superposition des filtres interdisent à l'archéologue toute lecture immédiate, un certain nombre d'observations font tout de même réfléchir. Ainsi, à Lunel-Viel, où l'étude anthropologique en cours ne révèle aucune différenciation par les caractères biométriques, la tombe 118 de la nécropole des Horts attire l'attention par son mobilier de parure abondant et caractéristique du costume barbare $\mathrm{du} \mathrm{VI}^{\mathrm{e}} \mathrm{s}$., avec une fibule circulaire, une fibule aviforme, des boucles d'oreilles de type nordique, une boucle de ceinture, un étui contenant un nécessaire de couture (Hernandez, 2001, p. 287-293). La série se distingue au sein de la nécropole et j'incline à voir dans un ensemble clos aussi démonstratif une sépulture extérieure au contexte local, une sépulture «barbare ». Une telle observation demeure exceptionnelle, mais il faut bien la rattacher à d'autres indices contemporains, comme les quelques noms germaniques mentionnés par l'épigraphie (Février, 1978b, p. 238). Épars, de tels indices mériteraient une étude d'ensemble qui en dirait long sur « la dispersion des populations barbares dans le Midi » (Février, 1978b, p. 239). Il faut gagner le Sud-Ouest pour observer des faits plus massifs, comme ces groupes de sépultures à Molandier et à L'Isle-Jourdain qui ont livré un mobilier en telle abondance et en tel décalage par rapport aux assemblages habituels, avec parfois des armes, que l'on est en droit d'envisager la présence de groupes francs (Stutz, 1996, p. 169; Bach, Boudartchouk, 1998). Mais pour quelques exemples aussi nets, on observe le plus souvent l'adoption progressive par la population locale de certains objets d'un type nouveau. Entre Septimanie et Novempopulanie, le contraste est frappant mais reste mal cerné, dans l'attente d'un inventaire plus complet qui semble à portée de main. Après des décennies de tâtonnements et d'excès "ethni-cistes ", la recherche doit sortir d'une phase de timidité stérilisante, où l'on n'osait plus avancer d'hypothèses. Celles-ci s'avèrent plus nécessaires que jamais, avec la garantie que, désormais, 
la masse documentaire interdit les interpré-tations hâtives. Par ses avancées, l'anthropologie biologique dont on avait longtemps attendu des réponses catégoriques doit aussi servir de modérateur.

\section{DE LA SÉPULTURE INDIVIDUELLE À LA SÉPULTURE MULTIPLE}

Partir seul ou bien accompagné? La question ne se pose pas réellement dans ces termes dans la mesure où l'on ne connaît aucun exemple d'inhumations multiples simultanées dans les nécropoles tardo-antiques de la Gaule méridionale. Si la sépulture individuelle reste la règle, une évolution s'ébauche néanmoins qui va conduire lentement de la sépulture unique aux réutilisations, processus largement observé à partir de l'Antiquité tardive (Crubézy, Raynaud, 1988).

Plutôt que de multiplier les citations hasardeuses sur des cas épars, intéressons-nous au site de Lunel-Viel où l'on dispose d'une population homogène que l'on suit un millénaire durant. Dans la nécropole du Verdier, la réutilisation d'une tombe demeure exceptionnelle dans les secteurs où dominent les tombes nord-sud, au début du $\mathrm{IV}^{\mathrm{e}}$ s., avec seulement 2 cas de réutilisation sur 83 tombes exhumées, soit 2,4\%. Cette pratique devient plus présente dans les tombes est-ouest au sein desquelles la proportion s'élève à $10 \%$, au V $\mathrm{V}^{\mathrm{e}}$., avant de décroître dans la zone nordouest, jusqu'à l'abandon de la nécropole. Puis la proportion s'élève encore jusqu'à $13 \%$ dans la nécropole des Horts, occupée aux $\mathrm{VI}^{\mathrm{e}}$ et $\mathrm{VII}^{\mathrm{e}} \mathrm{s}$., pour atteindre enfin 15 à $30 \%$ dans la nécropole Saint-Vincent, occupée sans discontinuer pendant tout le Moyen Âge.

Une série de variantes permet de suivre les étapes de la transformation, depuis la sépulture individuelle indemne de tout remaniement jusqu'à l'ossuaire dont on recense deux exemples seulement au Verdier, contenant jusqu'à cinq sujets ayant subi une ou plusieurs manipulations (fig. 77). De la sépulture primaire à la sépulture secondaire puis à l'ossuaire, à travers ces manipulations des restes humains émerge un lent changement d'attitude à l'égard des morts. Avant de poser la question de l'idée que l'on se faisait de la mort à la fin de l'Antiquité, il s'agit de comprendre ce que l'on faisait aux morts, matériellement, très concrètement, dans ce qui en subsistait et avec lesquels les vivants pouvaient entretenir un contact physique. Après avoir constaté la continuité des pratiques et l'ambivalence des techniques qui caractérisent la construction de la tombe ainsi que les gestes accompagnant les funérailles, dont la

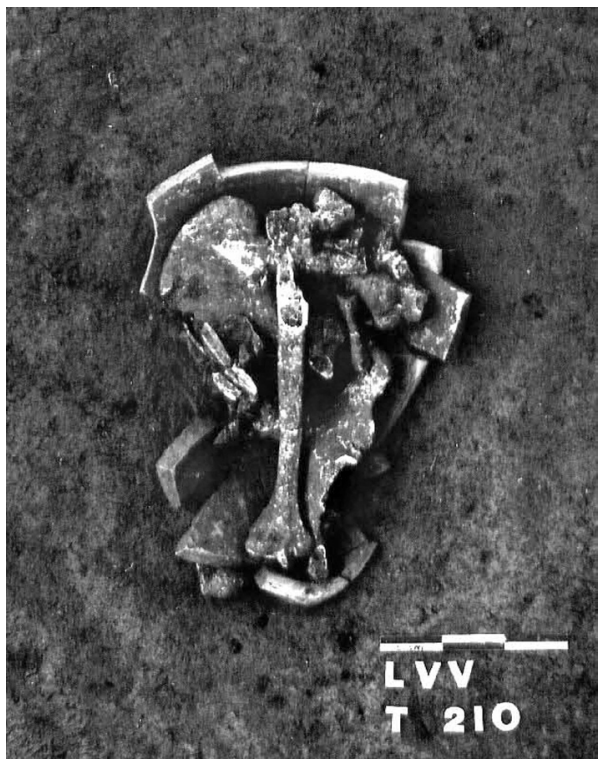

Fig. 77 - Le Verdier à Lunel-Viel (Hérault). Tombe 210 : réduction dans une amphore-ossuaire du $V^{e}$ s. (cliché C. Raynaud, CNRS).

stabilité déjoue l'attente de l'historien à l'égard des signes de changement, la manipulation du mort, sa réduction et sa réinhumation n'apparaissent-ils pas décisifs au sein du processus dont on suit le développement?

Martelons ce fait qu'au-delà de la diversité des aménagements et de l'éventail du choix des offrandes, le caractère dominant des tombes de l'Antiquité tardive réside dans leur utilisation individuelle. Une fois le mort déposé dans la tombe, les vivants s'attachent à respecter l'intégrité de la fosse devenue locus religiosus. Partout s'affirme le soin attaché à ne pas exhumer ou détériorer les sépultures anciennes, à la campagne comme à la ville (Carru dir., 1991, p. 15 et 28). Sans aller jusqu'à invoquer la loi sacrée en vertu de laquelle on tenait les morts à l'écart des vivants, à Rome, il faut insister sur la forte croyance dans le double du mort, présent dans la tombe et prêt à défendre l'intégrité de sa dernière demeure en revenant tourmenter les vivants (Jobbé-Duval, 1924). C'est cette crainte du retour du mort malfaisant qui explique, mieux que tout autre contrainte y compris juridique, que l'on ne réutilise pas la tombe. Il ne faut pas y toucher afin de se concilier les lémures, ou à tout le moins afin de tenir les morts à l'écart et d'éviter les troubles qu'ils peuvent causer. Cette prévention va jusqu'à proscrire tout contact avec le mort impur, répulsion qui traverse la mentalité antique et atteint à l'horreur charnelle, encore à la fin de l'Antiquité. En Gaule, plusieurs conciles condamnent, $\mathrm{au} \mathrm{VI}{ }^{\mathrm{e}} \mathrm{s}$, l'acte impie qui consiste à réutiliser 
une sépulture, usurpant un domaine sacré : s'il y avait lieu de réitérer l'interdit, c'est donc que la pratique progressait, mais restait condamnable. Il fallait insister sur ce contexte mental et sur cette sensibilité à l'égard des morts pour mesurer la portée des premiers indices d'évolution, puis leur multiplication dans les nécropoles. D'abord accidentelle, la réouverture de la tombe est ensuite pratiquée en vue d'une nouvelle sépulture. Dans un premier temps limitée à un seul sujet, la réutilisation se fait ensuite plus fréquente et peut regrouper deux à quatre sépultures dans la même tombe. Évolution lente, dont les étapes sont difficiles à situer dans la mesure où, d'une part, les sépultures restent mal datées et, d'autre part du fait que le changement ne fut probablement pas linéaire. Tout ce que l'on en perçoit tient à la chrono-logie relative, lorsqu'une sépulture recouvre, perturbe ou remplace celle qui l'avait précédée dans la tombe. Malgré leur ténuité, ces liens stratigraphiques nous renseignent sur une évolution qui va dans le sens d'une progressive atténuation de la crainte des morts : dans toutes les nécropoles fouillées, entre les premières tombes et les plus récentes il y a bien changement, même si le rythme et la chronologie en demeurent incertains.

Le type de la tombe n'était peut-être pas étranger à sa réutilisation, soit que celle-ci eût été envisagée d'emblée, soit qu'elle fut favorisée par la disposition matérielle. La question se pose particulièrement à propos des sarcophages, par exemple à Ménerbes où trois des vingt-huit sarcophages seulement échappèrent aux inhumations successives, sans que l'on connaisse le rythme des réutilisations sur un site où l'aire funéraire persista jusqu'au Moyen Âge (Cartron et al., 1992).

L'opposition entre sépulture primaire et sépulture secondaire fonde une large part de l'analyse des pratiques funéraires, sans pour autant épuiser l'écheveau complexe des situations et des solutions adoptées. Entre la sépulture primaire des funérailles et la sépulture secondaire, ultime lieu de dépôt après parfois plusieurs manipulations, intervient une série de gestes par lesquels est négocié le déplacement du défunt. Car, au début, ce déplacement n'est pas évident, il est même porteur de crainte pour qui l'ordonne et pour qui le réalise. Pour suivre le processus, il faut s'attacher à voir comment s'effectue la réutilisation de la tombe, selon que l'on évite tout contact ou toute manipulation de la première dépouille ou que l'on consente au contraire à une réduction partielle ou totale du squelette, avant d'aller dans les cas extrêmes jusqu'au déplacement des os vers une sépulture secondaire ou vers l'ossuaire. Entre corps en place et ossements déplacés, l'opposition n'est pas simplement binaire car interviennent des cas mixtes montrant qu'il s'agissait de limiter les effets de la réutilisation. On le voit dans les cas où le corps du premier occupant de la tombe n'est que partiellement remanié pour faire place à une nouvelle sépulture : les membres inférieurs sont repoussés vers le bord du coffre tandis que l'on prend soin de ne pas déplacer le tronc ni la tête, siège de la personnalité. Parlera-t-on alors de sépulture primaire localement remaniée, de réduction partielle? Dans la gradation des gestes afférents à la réutilisation des tombes, transparaît la façon dont fut négociée cette réduction de l'individualité du mort que, déjà, les vivants poussaient vers l'oubli. Vers l'oubli ou vers une autre forme du souvenir? À ces questions, l'archéologie ne peut apporter de réponse, mais elle suggère une tendance : longtemps contenus dans la fosse de la sépulture primaire, les morts peu à peu perdent leur lien avec le locus memoriae. L'évolution au demeurant n'est pas datée et les variantes que l'on veut ranger dans une progression échappaient peut-être à cette chronologie. Cet effacement du mort individuel, doit-on l'interpréter de façon négative, en terme d'oubli, peut-on penser au contraire à un regroupement familial perpétuant le lieu de la mémoire?

Ces pratiques témoigneraient donc d'une attitude nouvelle face à la tombe qui semble de moins en moins perçue comme un lieu inviolable mais devient insensiblement un lieu de passage. Ainsi poserai-je enfin la question de la christianisation.

\section{LA CHRISTIANISATION DES NÉCROPOLES : UNE BONNE QUESTION ?}

Les archéologues ont longtemps scruté les nécropoles de l'Antiquité tardive pour y lire les signes d'une christianisation, en ville comme à la campagne. Pourtant, Sidoine Apollinaire a éveillé notre attention à l'égard de ces signes, si vite effacés autour de la tombe du grand-père chrétien, si ostensibles mais si peu «chrétiens» autour de celle du consul Syagrius. L'archéologie dit-elle autre chose ? Au fil des thèmes évoqués, depuis la topographie funéraire jusqu'au mobilier des offrandes, de l'orientation à la construction de la tombe, nous avons vu s'effacer ou s'affaiblir les repères habituellement retenus à l'égard des progrès de la christianisation. P.-A. Février avait maintes fois réitéré la mise en garde contre une lecture trop littérale de ces gestes qui empruntaient aux pratiques sociales autant ou plus qu'à une religion qui, on le sait, ne s'occupa que fort tardivement d'encadrer le passage des morts. 
$\mathrm{Au} \mathrm{IV}^{\mathrm{e}} \mathrm{s}$. encore, le mobilier funéraire ne se distingue pas radicalement de ses antécédents du Haut-Empire, si ce n'est par sa quantité décroissante, jusqu'à sa disparition progressive au $\mathrm{V}^{\mathrm{e}} \mathrm{s}$. Certains attributs traversent les âges, comme la lampe, symbole de lumière éternelle, qui achève $\mathrm{au} \mathrm{IV}^{\mathrm{e}} \mathrm{s}$. un millénaire de service funèbre avant de disparaître au $\mathrm{V}^{\mathrm{e}} \mathrm{s}$. De cette longue coexistence entre tradition païenne et expérience chrétienne, témoigne encore le fréquent réemploi de sarcophages à thèmes mythologiques, où vient s'ajouter une épitaphe chrétienne, comme sur le fameux sarcophage d'Innodius, à la Gayole. L'exemple est connu et ressort d'une formation intellectuelle des élites, dans laquelle le partage n'est pas fait avec la culture païenne. Je ne l'ai mentionné que pour insister sur l'impossibilité de trancher fermement entre des signes païens et des signes chrétiens, tout au moins dans ce qui parvient à l'archéologue, dans les vestiges matériels. À Lunel-Viel, où les nécropoles tardo-antiques ont été fouillées quasiment in extenso, c'est seulement au $\mathrm{VI}^{\mathrm{e}} \mathrm{s}$. qu'apparaissent les premiers signes indiscutables d'une christianisation de la sépulture avec les deux stèles découvertes dans la nécropole des Horts (Février, Leyge dir., 1986, p. 115-116). L'une d'entre elles était implantée sur un édicule, rustique memoria marquant une tombe dont la sépulture se trouva réduite, les ossements rassemblés dans une fosse en position secondaire, probablement lors de l'abandon de la nécropole, au $\mathrm{VII}^{\mathrm{e}} \mathrm{s}$. (fig. 78). Au même moment une seconde aire funéraire se développait autour de l'emplacement de l'église SaintVincent, que divers indices stratigraphiques invitent à voir construite au $\mathrm{VI}^{\mathrm{e}} \mathrm{s}$. (Raynaud dir., à paraître a et b).

Ailleurs, les signes de christianisation demeurent bien rares, comme ces croix pattées laissées en réserve sur deux sarcophages languedociens, l'un dans l'église SaintAndré à Agde, fameuse pour le concile wisigothique qui s'y tint au début du VI ${ }^{\mathrm{e}}$ s. (Houlès, 1987, p. 116), l'autre dans la nécropole Saint-Saturnin-de-Nodels, près d'Aimargues (Raynaud dir., 2004, p. 57), les deux étant malheureusement mal datés. En revanche, c'est au $\mathrm{VI}^{\mathrm{e}} \mathrm{s}$. que se placent les sarcophages de Saint-Estève à Ménerbes, évoqués plus haut et arborant une série exceptionnelle de signes chrétiens, marques funéraires qui ne laissent aucune ambiguïté (fig. 76). En ce lieu, la topographie témoignait tout autant d'une christianisation qui, malgré l'incertitude laissée par une fouille inachevée, ne laisse guère de doute quant à la présence d'une église funéraire, peut-être suscitée par l'attraction des premières sépultures (Cartron et al., 1995). Il apparaît donc clairement, une nouvelle fois, qu'hormis les rares cas de marquage explicite de la tombe,

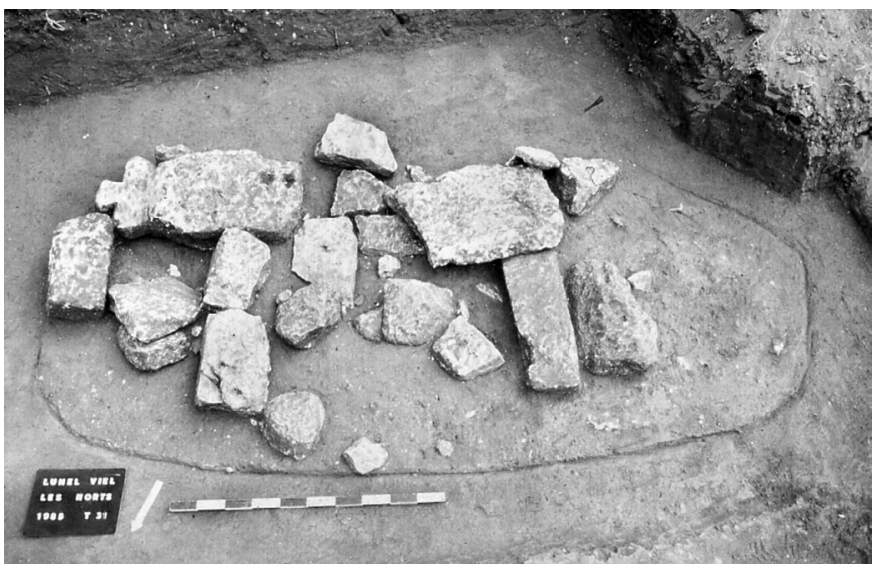

Fig. 78 - Les Horts à Lunel-Viel (Hérault). La tombe 31 de la nécropole transformée en memoria chrétienne. La stèle portant une croix se trouve en position d'effondrement, à l'angle supérieur gauche de la photo (cliché C. Raynaud, CNRS).

l'emprise de la religion demeure perceptible principalement dans la topographie des lieux funéraires dont certains - mais certains seulement, on l'a vu - se trouvent polarisés autour d'édifices du culte.

Le cas est banal et je ne m'y attarde guère, relevant seulement quelques éléments nouveaux apparus au cours de ces dernières années. Sur cette relation entre édifices chrétiens et sépultures, dont la succession demeure mal établie, les fouilles récentes ayant plutôt touché à des sites épiscopaux, à Narbonne (Ginouvez, 1999) ou à Maguelone (Barruol, Raynaud, 2002, p. 512), la documentation ne s'est guère étoffée en ce qui concerne le monde rural. À l'image du cas de Lunel-Viel évoqué plus haut, le site de Larina, à Hières-sur-Amby, révèle un processus analogue avec l'abandon de la première nécropole vers le début $\mathrm{du} \mathrm{VI}^{\mathrm{e}} \mathrm{s}$., au profit d'un nouveau champ funéraire autour de l'église, sur la colline du Mollard (Porte, 2001). Mais le cas n'est peut être pas général et l'on connaît aussi des lieux où l'église paléochrétienne ne semble pas avoir attiré les sépultures, à Pampelune où les fouilles récentes ont seulement mis au jour un petit groupe de tombes, près de l'église de l'agglomération perchée (Schneider, 2003), mais aussi à Loupian où l'église de Sainte-Cécile ne semble pas avoir davantage regroupé les morts (Duval dir., 1995). Dans ces cas, estce seulement la présence du baptistère qui a contribué à préserver les églises de l'envahissement funéraire? C'est possible, mais on peut aussi envisager en ces lieux d'autres nécropoles, à distance de l'aire cultuelle.

Ce qui ressort des observations nouvelles et ce que notait déjà P.-A Février, c'est que le regroupement des morts 
autour du sanctuaire, que celui-ci soit voué au culte ou qu'il soit d'abord funéraire, ne s'opère pas avant le $\mathrm{VI}^{\mathrm{e}} \mathrm{s}$., voire seulement au VII ${ }^{\mathrm{e}}$ s. (Février, 1978b, p. 237). Et peut-être doit-on insister sur un décalage longtemps proclamé et désormais avéré, entre la ville où l'église semble capter les tombes - certaines en tout cas - dès le $\mathrm{V}^{\mathrm{e}} \mathrm{s}$., et la campagne où la «capture » ne s'opère pas avant le milieu du $\mathrm{VI}^{\mathrm{e}} \mathrm{s}$, à Lunel-Viel et guère avant à Maguelone qui, sur son îlot lagunaire campe la figure insolite d'un évêché «à la campagne ». Pour l'essentiel donc, et malgré certains signes avant-coureurs, la topographie funéraire demeure jusqu'à la fin de l'Antiquité dans le droit fil des pratiques les plus anciennes. Plus tard viendra le temps du cimetière chrétien lorsque l'église, rompant avec son conformisme social, étendra son emprise sur les pratiques funéraires, mais ce ne sera pas avant l'an mil (Garnotel, Raynaud, 1996). 